\title{
Effects of starter culture and storage on volatile profiles and sensory characteristics of yogurt or cream butter
}

\section{Engin Gundogdu', Songul Cakmakci ${ }^{2 *}$, Ali Adnan Hayaloglu}

\footnotetext{
${ }^{1}$ Gümüşhane University, Department of Food Engineering, 29100 Gümüşhane, Turkey

${ }^{2}$ Atatürk University, Department of Food Engineering, 25240 Erzurum, Turkey

${ }^{3}$ Inönü University, Department of Food Engineering, 44280 Malatya, Turkey

*Corresponding author: songulcakmakci@hotmail.com; cakmakci@atauni.edu.tr
}

\section{Abstract}

In this study volatile compounds and sensory properties of butter produced from cream or yogurt using Lactococcus lactis subsp. lactis biovar. diacetylactis or Leuconostoc mesenteroides subsp. cremoris as well as a mixture of these two bacteria were investigated over 60 days. A total of 10 esters, 6 aldehydes, 11 ketones, 16 alcohols, 6 acids, 2 sulphides, 3 terpenes, and 3 miscellaneous compounds were detected. There were more volatile compounds in cream butter than in yogurt butter. While S-methyl thioacetate was only found in yogurt butter samples, hexanal, ethanol, 2-nonanone, 2-pentanone, 2-heptanone, acetic, butanoic (butyric) and hexanoic acid were the most abundant volatiles in both cream and yogurt butter. The results showed that the use of starter in the manufacturing of yogurt butter, which is traditionally produced without starters, affected the volatile compounds and sensory properties. The highest general acceptability scores were given to the butter samples containing mixed cultures at the end of storage.

Key words: yogurt butter, cream butter, starter culture, volatiles, sensory properties, storage

\section{Introduction}

Worldwide, butter is produced from cream, while in Turkey it is produced either from cream or from yogurt. Yogurt butter is called "yayık butter", which is famous for its pleasant flavour. Yayık, a wooden barrel, was used traditionally in the rural areas for the churning of yogurt (Sağdiç et al., 2002). Currently, electrical machines are also used to produce butter (Şenel, 2010). In fact, when yogurt is used as a raw material, it is called "yogurt butter". Yogurt butter is produced for traditional consumption in a small scale, but excess consumption is sold at village and local markets. In recent years, consumers have given more importance to traditional products (Altundas and Korukluoglu, 2019). Traditional foods are maintained by documentation of protected geographical indication (PGI) and protected designation of origin (PDO) which recognized by the European Union and these were distinguished from other industrial products (Mallia et al., 2005). From this perspective, the chemical, physical and microbiological properties of these products should be determined to prepare standards and explore their best characteristics. Some investigations were performed on the fatty acid profiles of yogurt butter 
(Sağdıç et al., 2004), the storage stability of yayık butter (Ayar et al., 2010), oxidative and lipolytic stabilities of yayık butter (Şenel et al., 2011) and comparison of cream and yogurt butter (HayaloğIu and Konar, 2001; Sağdıç et al., 2004). However, none of the authors compared these two butter types in terms of their volatile profiles during storage. For this purpose, we aimed to compare two types of butter made from yogurt or cream in terms of the volatiles determined by solid phase-micro extraction (SPME) and coupled with the gas chromatography-mass spectrometry (GC-MS) method. One type of butter from cream is commonly produced in Turkey whereby the cultures and its production are well-known. However, the other type of butter made from yogurt needs to be studied in terms of revealing its production methodology and effects of starter cultures on the final product. In this context, yogurt cultures, L. lactis subsp. lactis biovar. diacetylactis 4366 and Leu. mesenteroides subsp. cremoris 20200 or their mixture on the volatile profiles and sensory properties of the butter types were tested during storage.

\section{Materials and Methods}

\section{Materials}

Raw cow milk was obtained from a pilot dairy plant (Atatürk University, Agricultural Faculty, Erzurum, Turkey). DVS YC350 (Streptococcus thermophilus and Lactobacillus delbrueckii subsp. bulgaricus) used for yogurt production was obtained from Chr. Hansen, Istanbul, Turkey. For cream butter manufacture, cream was obtained from LEBEN Dairy Products A.Ş. (Erzurum, Turkey). Starters for cream fermentation were $L$. lactis subsp. lactis biovar. diacetylactis 4366 and Leu. mesenteroides subsp. cremoris 20200, which were purchased from DSMZ (Deutsche Sammlung von Mikroorganismen und Zellkulturen $\mathrm{GmbH}$, Braunschweig, Germany).

\section{Activation of starter cultures}

M17 broth for $L$. lactis subsp. lactis biovar. diacetylactis and MRS broth for Leu. mesenteroides subsp. cremoris were used. Both starter cultures were inoculated in test tubes containing sterilized MRS (at $30{ }^{\circ} \mathrm{C}$ for $24-48 \mathrm{~h}$ under anaerobic conditions) and $\mathrm{M} 17$ (at $30^{\circ} \mathrm{C}$ for $24-48 \mathrm{~h}$ ) broths. Then, $0.1 \mathrm{~mL}$ of broth was incubated on M17 and MRS agar plates at the same conditions mentioned above. Subsequently, the colonies were transferred from plates into tubes containing sterile saline water $(0.85 \%)$ and were compared with the McFarland scale 5 (McFarland Standard, Ref. 70 900, BioMérieux ${ }^{\oplus}$ ). Then, $1 \mathrm{~mL}$ of the sterile saline solution containing $10^{7} \mathrm{cfu} / \mathrm{mL}$ organisms was transferred to a sterilized container with a $10 \%$ dry-matter fat-free milk powder. The incubation was stopped when the acidity reached 0.36-0.39 \% and was used for butter production.

\section{Yogurt butter production}

Butter was produced after conversion of milk into yogurt. For yogurt production, raw milk was standardized to $5 \%$ fat content and heated to $90{ }^{\circ} \mathrm{C}$ for 20 min. After cooling to $43 \pm 7^{\circ} \mathrm{C}$, yogurt starter was added at a level of $2 \%(\mathrm{v} / \mathrm{v})$ and incubated until a $\mathrm{pH}$ of $4.7 \pm 1$ before it was immediately cooled to $21 \pm 7^{\circ} \mathrm{C}$ in ice water. The sample was then divided into four parts. One part was a starter-free butter used as a control (YA). The remaining three parts were inoculated with $L$. lactis subsp. lactis biovar. diacetylactis (YB), Leu. mesenteroides subsp. cremoris (YC) or a mixture of L. lactis subsp. lactis biovar. diacetylactis + Leu. mesenteroides subsp. cremoris (YD) at a level of $2 \%(\mathrm{v} / \mathrm{v})$. All groups were ripened overnight at $21 \pm 1{ }^{\circ} \mathrm{C}$ and viable cell counts in the starter cultures were approximately $10^{7} \mathrm{cfu} / \mathrm{mL}$. Subsequently, water was added into yogurt at its $50 \%$ by volume and churned using a mechanized device at $17-18^{\circ} \mathrm{C}$ until butter granules formed. After formation of butter, it was worked to obtain a homogenous butter granules and to form a continuous fat phase containing a finely dispersed water phase (no visible water). Approximately $100 \mathrm{~g}$ of the samples were wrapped with polyethylene bags and covered with aluminium foil to prevent light exposure before storage at $4 \pm 1^{\circ} \mathrm{C}$ for 2 months.

\section{Cream butter production}

The cream ( $\mathrm{pH} \mathrm{6.60)} \mathrm{containing} 50 \%$ milk fat was pasteurized at $85^{\circ} \mathrm{C}$ for 5 min and then rapidly 
cooled to $21 \pm 1{ }^{\circ} \mathrm{C}$ and divided into four aliquots. One part was starter-free butter (CA). The remaining three parts were inoculated with L. lactis subsp. lactis biovar. diacetylactis (CB), Leu. mesenteroides subsp. cremoris (CC) or the mixture of L. lactis subsp. lactis biovar. diacetylactis plus Leu. mesenteroides subsp. cremoris (CD). The viable cell counts in the starter cultures were at approximately $10^{7} \mathrm{cfu} / \mathrm{mL}$ in cream samples. Then, all samples were ripened overnight at $21 \pm 1^{\circ} \mathrm{C}$ until the $\mathrm{pH}$ reached $5 \pm 1$, churned at $14-16^{\circ} \mathrm{C}$, washed twice with cold water at $13-15^{\circ} \mathrm{C}$ and then worked to obtain a homogenous butter granules and to form no visible water. Packaging and storage conditions were the same as the yogurt butter samples.

\section{Volatiles in butter samples}

The volatile analysis in butter was performed using a Shimadzu GC-2010 gas chromatographyQP-2010 mass spectrometry system (Shimadzu Corporation, Kyoto, Japan) and run in the split (ratio was 1:20) mode. For this aim, $3.0 \mathrm{~g}$ of frozen $\left(-26{ }^{\circ} \mathrm{C}\right)$ butter samples were transferred into a 15-mL glass vial (Supelco, Bellefonte, PA, USA) and $10 \mu \mathrm{L}$ of internal standard 2-methyl-3-heptanone in methanol (Sigma-Aldrich Co., Taufkirchen, Germany) was added, which was left at $40{ }^{\circ} \mathrm{C}$ for 30 min. Extraction of volatiles was performed using a solventless technique (Pawliszyn et al., 1997). Extraction was achieved by injecting a $75-\mathrm{mm}$ carboxen-polydimethylsiloxane (CARPDMS) fibre (Supelco, Bellefonte, PA, USA) into the vial and exposing it to the headspace for $30 \mathrm{~min}$ at $40^{\circ} \mathrm{C}$. The fibre was positioned at 3.0 scale units in each run. Helium was used as the carrier gas at a flow rate of $1.0 \mathrm{~mL} / \mathrm{min}$ and a DB-Wax column (60 $\mathrm{m} \times 0.25$ $\mathrm{mm} \times 0.25, \mu \mathrm{m})$ (J\&W Scientific, Folsom, CA, USA) was used for the separation of volatile compounds. The oven was held at $40{ }^{\circ} \mathrm{C}$ for 2 min which was raised to $240{ }^{\circ} \mathrm{C}$ at a rate of $5{ }^{\circ} \mathrm{C}$ per minute and hold at this temperature for 5 minutes. The total analysis time for one sample was 47 minutes. The mass spectrometer was adjusted at 33-450 amu (threshold 1000) at a sampling rate of 1.11 scans/s. Before relative calculation of the volatile compounds in butter samples, retention index (RI) at the same conditions was identified using n-alkane series $C_{10}-C_{26}$ (Sigma-Aldrich Co., Taufkirchen, Germany) and was compared with those in http://webbook.nist.gov/chemistry/. The relative amounts of volatiles were calculated by the comparison of the peak area of the internal standard and unknown compounds. Each compound was expressed as micrograms per $100 \mathrm{~g}$ of sample.

\section{Sensory analysis}

The sensory properties of the butter samples were evaluated using the method described by Çakmakçı et al. (2014) and the sensory criteria were chosen according to the characteristics of yogurt and cream butters. The sensory properties of the butter samples by a panel consisting of eight panelists (ages 20-50-years old) who were experienced and familiar with yogurt, cream and butter flavour. They were no smoking and also volunteer for sensory analysis. The samples (about 50 g, wrapped with aluminium foil and stored at $4{ }^{\circ} \mathrm{C}$ ) were used for sensory analysis on 1, 15, 30, 45 and 60 days of storage. The tests were conducted at Food Engineering (Atatürk University) department laboratory, by students and teaching staff. Six parameters including colour, texture, odour, flavour, rancid taste and general acceptability were evaluated. Scores varied on a scale of 1 (poor) to 9 points (excellent). The tests were conducted at florescence light. Warm water and plain bread were used to rinse their palates between butter samples.

\section{Statistical analysis}

The experimental design was based on a completely randomized factorial design as follows: two materials (cream and yogurt butter) $\times$ four starter culture systems (control without culture, $L$. lactis subsp. lactis biovar. diacetylactis, Leu. mesenteroides subsp. cremoris, L. lactis subsp. lactis biovar. diacetylactis + Leu. mesenteroides subsp. cremoris $\times$ five storage times (1, 15, 30, 45 and 60 days) and two replicates. The SPSS program, version 13.013 .0 for Windows (SPSS Inc., Chicago, IL, USA) was used for statistical analysis of the data using the analysis of variance (ANOVA). Duncan's multiple range tests were used to compare the means. 
Additionally, PCA analysis was applied to the data from GC-MS of volatile compounds to simplify and graphically assess the changes between butter samples.

\section{Results and discussion}

\section{$\mathrm{pH}$ and chemical composition}

Table 1 shows $\mathrm{pH}$ values and chemical composition of the butter samples. The $\mathrm{pH}$ values in the samples ranged from 4.02 (sample YA) to 5.65 (sample CA). Differences in the $\mathrm{pH}$ of yogurt and cream butters samples were significant $(P<0.01)$. It is most likely that the use of different types of starter bacteria also changed the $\mathrm{pH}$ of butter samples $(P<0.01)$. The same differences were also observed in the acidity (as percentage of lactic acid) levels of samples (Table 1). Hayaloglu and Konar (2001) compared more than twenty-five yogurt or cream butter from retails and the authors pointed out that the butter samples made from yogurt contained a higher level of acidity and lower levels of $\mathrm{pH}$ than those of butter samples made from cream. This may be due to the production of lactic acid during fermentation of yogurt by yogurt starter bacteria. Butters made from cream had higher fat, total solids and fat-free total solids than yogurt samples.

\section{Volatile composition}

In this study, a total of 61 volatiles were determined in both butter types. These volatiles were 10 esters, 6 aldehydes, 11 ketones, 16 alcohols, and 6 acids and are given in Tables 2 to 5 Additionally, 8 other compounds were identified.

TABLE 1. PH and chemical characteristics of fresh butter made from yogurt and cream

\begin{tabular}{|c|c|c|c|c|c|c|c|c|}
\hline \multirow[b]{2}{*}{ Variants } & \multicolumn{8}{|c|}{ Butters } \\
\hline & YA (Control) & YB & YC & YD & CA & CB & CC & $C D$ \\
\hline Total solid (\%) & $81.80 \pm 0.33^{B}$ & $80.55 \pm 0.81^{A}$ & $81.04 \pm 0.75^{\mathrm{AB}}$ & $81.07 \pm 0.74^{\mathrm{AB}}$ & $85.82 \pm 0.08^{D}$ & $83.67 \pm 0.12^{c}$ & $85.33 \pm 0.95^{D}$ & $83.82 \pm 0.33^{c}$ \\
\hline Fat (\%) & $80.63 \pm 0.19^{\mathrm{B}}$ & $79.56 \pm 0.43^{A}$ & $79.56 \pm 0.66^{A}$ & $80.19 \pm 0.47^{\mathrm{AB}}$ & $82.13 \pm 0.25^{c}$ & $80.69 \pm 0.69^{\mathrm{B}}$ & $82.25 \pm 0.29^{c}$ & $80.50 \pm 0.41^{\text {B }}$ \\
\hline Fat-free total solids & $1.18 \pm 0.14$ & $0.99 \pm 43$ & $1.48 \pm 0,61$ & $0.89 \pm 0.38$ & $3.69 \pm 0.33$ & $2.98 \pm 0.58$ & $3.08 \pm 0.71$ & $3.32 \pm 0.47$ \\
\hline $\mathrm{pH}$ & $4.02 \pm 0.00^{A}$ & $4.07 \pm 0.09^{A B}$ & $4.10 \pm 0.05^{B}$ & $4.07 \pm 0.03^{\mathrm{AB}}$ & $5.65 \pm 0.01^{\mathrm{E}}$ & $4.67 \pm 0.01^{c}$ & $5.56 \pm 0.05^{D}$ & $4.67 \pm 0.01^{c}$ \\
\hline Acidity (\% la) & $0.41 \pm 0.03^{A B C}$ & $0.53 \pm 0.03^{c}$ & $046 \pm 0.01^{B C}$ & $0.42 \pm 0.01^{B C}$ & $0.38 \pm 0.50^{A B C}$ & $0.23 \pm 0.01^{\mathrm{AB}}$ & $0.13 \pm 0.00^{A}$ & $0.23 \pm 0.01^{A B}$ \\
\hline
\end{tabular}

YA: yogurt butter control; YB: yogurt butter produced with L. lactis subsp. lactis biovar. diacetylactis (2 \%); YC: yogurt butter produced with Leu. mesenteroides subsp. cremoris (2\%); YD: yogurt butter produced with Leu. mesenteroides subsp. cremoris (1 \%) + L. lactis subsp. lactis biovar. diacetylactis (1\%); CA: cream butter control; CB: cream butter produced with L. lactis subsp. lactis biovar. diacetylactis (2 \%); CC: cream butter produced with Leu. mesenteroides subsp. cremoris (2\%); CD: cream butter produced with Leu. mesenteroides subsp. cremoris (1\%) + L. lactis subsp. lactis biovar. diacetylactis (1\%).

${ }^{A-E}$ :Values in the same row having different superscripts differ significantly $(\mathrm{P}<0.05)$. 
TABLE 2. Esters in butters made from yogurt and cream during 60 days of storage $(\mu \mathrm{g} / 100 \mathrm{~g})$

\begin{tabular}{|c|c|c|c|c|c|c|c|c|c|c|c|c|c|}
\hline \multirow[b]{2}{*}{ Volatiles } & \multirow[b]{2}{*}{ RI } & \multirow[b]{2}{*}{ Days } & \multicolumn{8}{|c|}{ Types of butter } & \multicolumn{3}{|c|}{ Statistics, Probability } \\
\hline & & & YA & YB & YC & YD & CA & $\mathrm{CB}$ & $\mathrm{CC}$ & $C D$ & $\mathrm{RM}$ & SC & ST \\
\hline \multirow{3}{*}{$\begin{array}{l}\text { Methyl } \\
\text { acetate }\end{array}$} & \multirow[t]{3}{*}{826} & 1 & ND & $0.9 \pm 0.6$ & $0.6 \pm 0.0$ & $0.6 \pm 0.0$ & $0.6 \pm 0.4$ & $1.5 \pm 1.4$ & $1.3 \pm 0.7$ & $1.0 \pm 0.6$ & 0.00 & 0.00 & 0.00 \\
\hline & & 30 & $1.4 \pm 0.5$ & $1.2 \pm 0.3$ & $1.5 \pm 0.9$ & $0.7 \pm 0.4$ & $1.6 \pm 2.2$ & $0.4 \pm 0.0$ & $8.2 \pm 2.5$ & $1.5 \pm 0.2$ & & & \\
\hline & & 60 & $0.5 \pm 0.0$ & ND & $1.0 \pm 0.4$ & $0.6 \pm 0.0$ & $1.0 \pm 0.0$ & ND & $3.6 \pm 0.0$ & $0.3 \pm 0.1$ & & & \\
\hline \multirow[t]{3}{*}{ Ethyl acetate } & \multirow[t]{3}{*}{887} & 1 & $10.6 \pm 9.6$ & $1.6 \pm 1.5$ & $4.3 \pm 3.5$ & $1.2 \pm 0.5$ & $2.4 \pm 1.0$ & $4.0 \pm 3.5$ & $13.6 \pm 5.6$ & $5.2 \pm 5.2$ & 0.00 & 0.00 & 0.00 \\
\hline & & 30 & $2.9 \pm 1.7$ & $2.2 \pm 1.4$ & $3.2 \pm 0.0$ & $1.6 \pm 0.6$ & $251.2 \pm 83.3$ & $131.0 \pm 45.3$ & $232.4 \pm 86.8$ & $175.1 \pm 27.8$ & & & \\
\hline & & 60 & $1.4 \pm 0.5$ & $0.4 \pm 0.2$ & $1.5 \pm 0.8$ & $3.3 \pm 3.4$ & $358.5 \pm 7.9$ & $1.0 \pm 11.1$ & $363.7 \pm 150.4$ & $126.1 \pm 41.6$ & & & \\
\hline \multirow{3}{*}{$\begin{array}{l}\text { 2-methyl- } \\
\text { ethyl } \\
\text { propanoate }\end{array}$} & \multirow[t]{3}{*}{966} & 1 & ND & ND & ND & ND & $0.1 \pm 0.0$ & ND & $0.1 \pm 0.0$ & ND & 0.00 & 0.00 & 0.001 \\
\hline & & 30 & ND & ND & ND & ND & $14.6 \pm 6.9$ & ND & $16.3 \pm 6.3$ & ND & & & \\
\hline & & 60 & ND & ND & ND & $0.1 \pm 0.0$ & $14.9 \pm 1.4$ & ND & $7.9 \pm 7.7$ & ND & & & \\
\hline \multirow{3}{*}{$\begin{array}{l}\text { Methyl } \\
\text { butanoate }\end{array}$} & \multirow[t]{3}{*}{985} & 1 & $1.7 \pm 0.0$ & $3.5 \pm 3.6$ & $1.2 \pm 0.0$ & $5.7 \pm 2.9$ & $2.8 \pm 2.2$ & $1.4 \pm 0.0$ & $2.9 \pm 2.4$ & $1.7 \pm 0.0$ & 0.001 & 0.438 & 0.096 \\
\hline & & 30 & $7.4 \pm 1.7$ & $7.2 \pm 0.0$ & $7.1 \pm 2.3$ & $3.7 \pm 3.0$ & ND & ND & $1.9 \pm 0.5$ & $0.4 \pm 0.0$ & & & \\
\hline & & 60 & ND & ND & ND & $3.3 \pm 0.0$ & $36.0 \pm 14.7$ & ND & ND & $3.0 \pm 2.7$ & & & \\
\hline \multirow{3}{*}{$\begin{array}{l}\text { Ethyl } \\
\text { butanoate }\end{array}$} & \multirow[t]{3}{*}{1036} & 1 & $0.1 \pm 0.0$ & $0.6 \pm 0.0$ & $0.4 \pm 0.0$ & $0.6 \pm 0.2$ & $1.0 \pm 0.3$ & $0.9 \pm 0.0$ & $2.3 \pm 0.7$ & $0.7 \pm 0.2$ & 0.00 & 0.00 & 0.00 \\
\hline & & 30 & $0.5 \pm 0.1$ & $0.3 \pm 0.0$ & $0.5 \pm 0.2$ & $0.3 \pm 0.1$ & $67.2 \pm 28.1$ & $8.7 \pm 6.2$ & $285.3 \pm 86.4$ & $4.3 \pm 0.8$ & & & \\
\hline & & 60 & $0.7 \pm 0.3$ & $3.9 \pm 5.4$ & $0.7 \pm 0.3$ & $0.4 \pm 0.1$ & $65.6 \pm 10.0$ & $6.7 \pm 2.8$ & $119.5 \pm 41.5$ & $4.4 \pm 0.4$ & & & \\
\hline \multirow{3}{*}{$\begin{array}{l}\text { S-methyl } \\
\text { thioacetate }\end{array}$} & \multirow[t]{3}{*}{1049} & 1 & $3.1 \pm 1.0$ & $1.3 \pm 0.3$ & $1.5 \pm 0.1$ & $1.3 \pm 0.7$ & ND & ND & ND & ND & 0.019 & 0.000 & 0.002 \\
\hline & & 30 & $1.5 \pm 0.4$ & $1.0 \pm 0.7$ & $1.4 \pm 0.4$ & $1.1 \pm 0.1$ & ND & ND & ND & ND & & & \\
\hline & & 60 & $0.8 \pm 0.4$ & $0.2 \pm 0.0$ & $0.9 \pm 0.2$ & $1.3 \pm 0.3$ & ND & ND & ND & ND & & & \\
\hline \multirow{3}{*}{$\begin{array}{l}\text { Ethyl } \\
\text { hexanoate }\end{array}$} & \multirow[t]{3}{*}{1236} & 1 & $0.2 \pm 0.0$ & $0.2 \pm 0.1$ & $0.2 \pm 0.1$ & $0.2 \pm 0.2$ & $0.1 \pm 0.0$ & ND & $0.4 \pm 0.0$ & ND & 0.000 & 0.000 & 0.000 \\
\hline & & 30 & $0.2 \pm 0.0$ & $2.1 \pm 2.7$ & $0.4 \pm 0.2$ & $0.2 \pm 0.2$ & $6.2 \pm 0.5$ & $1.2 \pm 0.7$ & $145.3 \pm 30.7$ & $12.0 \pm 1.2$ & & & \\
\hline & & 60 & $2.0 \pm 1.8$ & $0.7 \pm 0.2$ & $1.6 \pm 0.0$ & $0.2 \pm 0.1$ & $6.0 \pm 0.2$ & $1.5 \pm 0.0$ & $99.6 \pm 14.4$ & $7.0 \pm 0.8$ & & & \\
\hline \multirow{3}{*}{$\begin{array}{l}\text { Ethyl } \\
\text { octanoate }\end{array}$} & \multirow[t]{3}{*}{1439} & 1 & $1.4 \pm 1.0$ & $0.8 \pm 0.1$ & $0.7 \pm 0.3$ & $0.8 \pm 0.4$ & $0.6 \pm 0.0$ & $1.1 \pm 0.2$ & $0.7 \pm 0.1$ & $0.8 \pm 0.1$ & 0.000 & 0.000 & 0.000 \\
\hline & & 30 & $0.5 \pm 0.2$ & $1.2 \pm 0.9$ & $0.6 \pm 0.1$ & $0.4 \pm 0.1$ & $3.8 \pm 0.3$ & $1.9 \pm 1.9$ & $44.5 \pm 8.5$ & $5.6 \pm 0.8$ & & & \\
\hline & & 60 & $5.2 \pm 4.7$ & $3.0 \pm 1.9$ & $2.5 \pm 0.4$ & $2.0 \pm 0.9$ & $9.4 \pm 1.2$ & $6.5 \pm 0.6$ & $35.4 \pm 7.5$ & $4.8 \pm 0.0$ & & & \\
\hline \multirow{3}{*}{$\begin{array}{l}\text { Methyl } \\
\text { decanoate }\end{array}$} & \multirow[t]{3}{*}{1600} & 1 & $15.2 \pm 13.1$ & $7.4 \pm 1.2$ & $6.5 \pm 2.3$ & $8.1 \pm 5.5$ & $5.8 \pm 0.8$ & $9.0 \pm 2.1$ & $7.3 \pm 0.8$ & $7.0 \pm 1.1$ & 0.146 & 0.485 & 0.022 \\
\hline & & 30 & $4.8 \pm 1.7$ & $3.5 \pm 1.6$ & $5.8 \pm 0.4$ & $3.6 \pm 0.7$ & $5.3 \pm 1.8$ & $4.1 \pm 0.5$ & $6.8 \pm 2.3$ & $4.5 \pm 0.1$ & & & \\
\hline & & 60 & $7.4 \pm 4.3$ & $5.8 \pm 2.5$ & $5.9 \pm 0.4$ & $5.1 \pm 1.9$ & $14.2 \pm 4.7$ & $14.0 \pm 4.2$ & $5.6 \pm 0.3$ & $4.2 \pm 0.7$ & & & \\
\hline \multirow{3}{*}{$\begin{array}{l}\text { Methyl } \\
\text { dodecanoate }\end{array}$} & \multirow[t]{3}{*}{1809} & 1 & $2.4 \pm 2.3$ & $1.1 \pm 0.2$ & $1.0 \pm 0.4$ & $0.6 \pm 0.0$ & $0.8 \pm 0.2$ & $1.2 \pm 0.1$ & $1.1 \pm 0.2$ & $0.9 \pm 0.2$ & 0.087 & 0.904 & 0.029 \\
\hline & & 30 & $0.8 \pm 0.0$ & $0.5 \pm 0.0$ & $0.9 \pm 0.3$ & $0.5 \pm 0.1$ & $0.8 \pm 0.3$ & $0.6 \pm 0.0$ & $0.8 \pm 0.3$ & $0.5 \pm 0.1$ & & & \\
\hline & & 60 & $1.2 \pm 0.7$ & $0.9 \pm 0.3$ & $0.8 \pm 0.0$ & $0.7 \pm 0.2$ & $1.6 \pm 0.9$ & $1.5 \pm 0.0$ & $0.8 \pm 0.1$ & $0.6 \pm 0.1$ & & & \\
\hline $\begin{array}{l}\text { Total esters } \\
\text { (10) }\end{array}$ & & & 73.9 & 51.5 & 52.7 & 48.2 & 872.1 & 198.2 & 1407.3 & 372.2 & & & \\
\hline
\end{tabular}

RT: retention index; RM: raw material (yogurt and cream); SC: starter culture; ST: storage time (1, 30 and 60. day); YA: yogurt butter control; YB: yogurt butter produced with L. lactis subsp. lactis biovar. diacetylactis (2 \%); YC: yogurt butter produced with Leu. mesenteroides subsp. cremoris (2\%); YD: yogurt butter produced with Leu. mesenteroides subsp. cremoris (1 \%) + L. lactis subsp. lactis biovar. diacetylactis (1\%); CA: cream butter control; CB: cream butter produced with L. lactis subsp. lactis biovar. diacetylactis (2 \%); CC: cream butter produced with Leu. mesenteroides subsp. cremoris (2 \%); CD: cream butter produced with Leu. mesenteroides subsp. cremoris $(1 \%)+$ L. lactis subsp. lactis biovar. diacetylactis (1\%) 
TABLE 3. Aldehydes and ketones in butters made from yogurt and cream during 60 days of storage ( $\mu \mathrm{g} / 100 \mathrm{~g})$

\begin{tabular}{|c|c|c|c|c|c|c|c|c|c|c|c|c|c|}
\hline \multirow[b]{2}{*}{ Volatiles } & \multirow[b]{2}{*}{ RI } & \multirow[b]{2}{*}{ Days } & \multicolumn{8}{|c|}{ Types of butter } & \multicolumn{3}{|c|}{ Statistics, Probability } \\
\hline & & & YA & YB & YC & YD & CA & CB & CC & $C D$ & RM & SC & ST \\
\hline \multirow[t]{3}{*}{ Acetaldehyde } & \multirow[t]{3}{*}{745} & 1 & $7.8 \pm 3.3$ & $4.6 \pm 2.1$ & $4.5 \pm 0.5$ & $4.7 \pm 3.0$ & $1.2 \pm 0.5$ & $1.8 \pm 1.6$ & $0.8 \pm 0.3$ & $1.8 \pm 0.2$ & 0.546 & 0.000 & 0.147 \\
\hline & & 30 & $4.7 \pm 2.1$ & $3.4 \pm 2.4$ & $5.3 \pm 1.4$ & $3.3 \pm 2.3$ & $1.8 \pm 0.8$ & $1.7 \pm 0.1$ & $1.2 \pm 0.4$ & $2.2 \pm 0.3$ & & & \\
\hline & & 60 & $1.2 \pm 0.2$ & $1.1 \pm 0.4$ & $1.8 \pm 0.1$ & $3.7 \pm 0.2$ & $3.3 \pm 0.0$ & $4.3 \pm 0.5$ & $1.5 \pm 0.0$ & $2.2 \pm 0.1$ & & & \\
\hline \multirow[t]{3}{*}{ Butanal } & \multirow[t]{3}{*}{876} & 1 & ND & $0.2 \pm 0.0$ & $0.3 \pm 0.0$ & ND & ND & $0.1 \pm 0.0$ & ND & $0.1 \pm 0.0$ & 0.018 & 0.124 & 0.274 \\
\hline & & 30 & $0.3 \pm 0.2$ & $0.2 \pm 0.1$ & $0.3 \pm 0.0$ & ND & $2.3 \pm 2.5$ & $0.2 \pm 0.0$ & ND & ND & & & \\
\hline & & 60 & ND & ND & $0.2 \pm 0.0$ & $0.3 \pm 0.0$ & $3.8 \pm 3.2$ & ND & ND & ND & & & \\
\hline \multirow{3}{*}{$\begin{array}{l}\text { 3-methy } \\
\text { butanal }\end{array}$} & \multirow[t]{3}{*}{919} & 1 & ND & ND & ND & ND & $0.8 \pm 0.1$ & $0.3 \pm 0.0$ & ND & $0.2 \pm 0.0$ & 0.003 & 0.014 & 0.111 \\
\hline & & 30 & ND & ND & ND & ND & $18.2 \pm 13.6$ & $0.2 \pm 0.0$ & ND & $0.2 \pm 0.0$ & & & \\
\hline & & 60 & $0.5 \pm 0.0$ & $0.2 \pm 0.0$ & $0.2 \pm 0.1$ & $0.1 \pm 0.0$ & $5.5 \pm 1.1$ & $1.3 \pm 1.0$ & ND & $0.2 \pm 0.3$ & & & \\
\hline \multirow[t]{3}{*}{ Hexanal } & \multirow[t]{3}{*}{1083} & 1 & $2.2 \pm 0.8$ & $1.1 \pm 0.7$ & $1.7 \pm 1.1$ & $1.0 \pm 1.0$ & $2.8 \pm 0.6$ & $17.1 \pm 0.2$ & $1.5 \pm 0.4$ & $9.1 \pm 1.0$ & 0.000 & 0.000 & 0.000 \\
\hline & & 30 & $2.4 \pm 1.5$ & $1.7 \pm 1.3$ & $2.9 \pm 0.3$ & $1.8 \pm 0.2$ & $51.3 \pm 57.8$ & $17.4 \pm 0.8$ & ND & $10.0 \pm 0.3$ & & & \\
\hline & & 60 & ND & ND & $7.5 \pm 0.0$ & $3.7 \pm 2.8$ & $347.9 \pm 87.6$ & $116.0 \pm 0.0$ & ND & $9.7 \pm 3.5$ & & & \\
\hline \multirow{3}{*}{$\begin{array}{l}\text { 3-methy-2- } \\
\text { butanal }\end{array}$} & \multirow[t]{3}{*}{1202} & 1 & $2.8 \pm 0.8$ & $2.1 \pm 0.4$ & $1.8 \pm 0.4$ & $1.3 \pm 0.5$ & ND & $0.4 \pm 0.0$ & ND & $0.1 \pm 0.0$ & 0.820 & 0.000 & 0.000 \\
\hline & & 30 & $1.8 \pm 0.1$ & $1.7 \pm 0.8$ & $2.7 \pm 0.3$ & $1.6 \pm 0.6$ & ND & ND & ND & ND & & & \\
\hline & & 60 & ND & ND & ND & $1.8 \pm 0.0$ & ND & ND & ND & ND & & & \\
\hline \multirow[t]{3}{*}{ Benzaldehyde } & \multirow[t]{3}{*}{1540} & 1 & $1.1 \pm 0.9$ & $0.6 \pm 0.1$ & $0.7 \pm 0.1$ & $0.5 \pm 0.1$ & $0.3 \pm 0.2$ & $0.5 \pm 0.1$ & $0.4 \pm 0.0$ & $0.4 \pm 0.1$ & 0.016 & 0.927 & 0.007 \\
\hline & & 30 & $0.4 \pm 0.1$ & $0.4 \pm 0.1$ & $1.2 \pm 0.9$ & $0.7 \pm 0.0$ & $2.3 \pm 2.4$ & $0.7 \pm 0.2$ & $0.4 \pm 0.1$ & $0.5 \pm 0.1$ & & & \\
\hline & & 60 & $22.2 \pm 30.2$ & $3.8 \pm 2.9$ & $1.7 \pm 1.0$ & $1.5 \pm 1.1$ & $25.2 \pm 6.1$ & $4.2 \pm 0.3$ & $1.2 \pm 0.4$ & $0.7 \pm 0.2$ & & & \\
\hline \multicolumn{3}{|c|}{ Total aldehydes (6) } & 47.4 & 21.1 & 32.8 & 26.0 & 466.7 & 166.2 & 7.0 & 37.4 & & & \\
\hline \multicolumn{14}{|l|}{ Ketones } \\
\hline \multirow[t]{3}{*}{ 2-propanone } & \multirow[t]{3}{*}{815} & 1 & $38.8 \pm 32.4$ & $6.0 \pm 3.9$ & $22.0 \pm 25.2$ & $5.5 \pm 4.5$ & $4.8 \pm 0.4$ & $7.6 \pm 8.5$ & $2.8 \pm 1.2$ & $4.3 \pm 2.2$ & 0.031 & 0.475 & 0.002 \\
\hline & & 30 & $6.5 \pm 2.0$ & $4.9 \pm 3.1$ & $9.2 \pm 0.9$ & $4.9 \pm 1.6$ & $12.9 \pm 3.3$ & $2.2 \pm 0.7$ & $3.4 \pm 7.1$ & $2.9 \pm 0.5$ & & & \\
\hline & & 60 & $65.7 \pm 78.5$ & $31.2 \pm 23.8$ & $15.5 \pm 1.2$ & $9.4 \pm 5.9$ & $62.3 \pm 50.9$ & $183.7 \pm 106.8$ & $6.9 \pm 4.9$ & $3.1 \pm 1.5$ & & & \\
\hline \multirow[t]{3}{*}{ 2-butanone } & \multirow[t]{3}{*}{903} & 1 & $20.4 \pm 4.07$ & $14.0 \pm 0.6$ & $16.3 \pm 1.6$ & $15.8 \pm 9.2$ & $15.0 \pm 1.5$ & $15.0 \pm 4.5$ & $18.4 \pm 3.3$ & $10.6 \pm 7.9$ & 0.002 & 0.000 & 0.000 \\
\hline & & 30 & $17.2 \pm 4.8$ & $15.6 \pm 1.4$ & $11.5 \pm 0.2$ & $14.6 \pm 3.1$ & ND & $15.0 \pm 4.9$ & ND & $8.8 \pm 11.0$ & & & \\
\hline & & 60 & $33.4 \pm 15.9$ & $34.4 \pm 0.0$ & $20.2 \pm 3.9$ & $20.2 \pm 1.0$ & $12.2 \pm 6.7$ & $35.8 \pm 2.5$ & $10.2 \pm 14.4$ & $3.1 \pm 4.3$ & & & \\
\hline \multirow{3}{*}{$\begin{array}{l}2,3- \\
\text { butanedione } \\
\text { (diacetyl) }\end{array}$} & 973 & 1 & $15.8 \pm 6.7$ & $21.0 \pm 6.5$ & $28.1 \pm 7.6$ & $21.9 \pm 11.4$ & $5.2 \pm 0.1$ & $187.5 \pm 9.8$ & $4.1 \pm 0.8$ & $131.6 \pm 50.1$ & 0.000 & 0.000 & 0.000 \\
\hline & & 30 & $12.2 \pm 9.1$ & $21.2 \pm 12.9$ & $49.2 \pm 6.6$ & $26.8 \pm 0.2$ & $6.3 \pm 2.9$ & $186.6 \pm 13.1$ & $2.1 \pm 0.4$ & $308.9 \pm 71.3$ & & & \\
\hline & & 60 & $4.8 \pm 0.0$ & $5.1 \pm 0.0$ & $19.1 \pm 3.2$ & $36.2 \pm 20.1$ & ND & $52.9 \pm 0.0$ & ND & $225.8 \pm 16.5$ & & & \\
\hline 2-pentanone & 978 & 1 & $123.6 \pm 153.1$ & $10.0 \pm 6.9$ & $39.4 \pm 45.1$ & $11.0 \pm 8.7$ & $14.6 \pm 0.8$ & $71.8 \pm 0.0$ & $12.5 \pm 4.4$ & $32.4 \pm 0.0$ & 0.000 & 0.014 & 0.000 \\
\hline & & 30 & $15.5 \pm 7.1$ & $12.4 \pm 7.3$ & $25.1 \pm 3.1$ & $14.7 \pm 1.2$ & $140.8 \pm 44.6$ & $154.0 \pm 150.6$ & $30.5 \pm 7.8$ & & & & \\
\hline & & 60 & $368.5 \pm 39.7$ & $295.5 \pm 0.4$ & $186.8 \pm 0.6$ & $52.3 \pm 53.8$ & $533.4 \pm 206.9$ & $411.3 \pm 41.7$ & $418.5 \pm 223.6$ & $62.6 \pm 0.0$ & & & \\
\hline 2,3- & 1055 & 1 & $6.6 \pm 1.6$ & $4.7 \pm 0.0$ & $7.5 \pm 1.6$ & $4.1 \pm 0.8$ & $0.1 \pm 0.0$ & $0.8 \pm 0.4$ & $0.1 \pm 0.0$ & $0.6 \pm 0.1$ & 0.021 & 0.000 & 0.004 \\
\hline & & 30 & $4.2 \pm 1.8$ & $3.2 \pm 0.3$ & $8.5 \pm 3.2$ & 3. $1 \pm 0.6$ & ND & $0.6 \pm 0.1$ & ND & $1.2 \pm 0.1$ & & & \\
\hline & & 60 & $1.2 \pm 0.0$ & $0.8 \pm 0.0$ & $3.3 \pm 0.0$ & $4.1 \pm 2.6$ & ND & $1.1 \pm 0.0$ & ND & $1.9 \pm 1.0$ & & & \\
\hline
\end{tabular}


TABLE 3. (Continued)

\begin{tabular}{|c|c|c|c|c|c|c|c|c|c|c|c|c|c|}
\hline \multirow[b]{2}{*}{ Volatiles } & \multirow[b]{2}{*}{ RI } & \multirow[b]{2}{*}{ Days } & \multicolumn{8}{|l|}{ Types of butter } & \multicolumn{3}{|c|}{$\begin{array}{l}\text { Statistics, } \\
\text { Probability }\end{array}$} \\
\hline & & & YA & YB & YC & YD & CA & $\mathrm{CB}$ & $\mathrm{CC}$ & $C D$ & RM & $\mathrm{SC}$ & ST \\
\hline \multirow[t]{3}{*}{ 2-heptanone } & \multirow[t]{3}{*}{1185} & 1 & $76.8 \pm 17.3$ & $38.8 \pm 5.9$ & $41.5 \pm 0.0$ & $36.9 \pm 18.0$ & $43.6 \pm 0.9$ & $62.2 \pm 27.8$ & $48.7 \pm 7.2$ & $48.2 \pm 1.5$ & 0.050 & 0.230 & 0.000 \\
\hline & & 30 & $46.5 \pm 12.1$ & $39.4 \pm 15.6$ & $95.4 \pm 42.8$ & $45.9 \pm 4.2$ & $161.9 \pm 59.2$ & $132.9 \pm 96.2$ & $94.5 \pm 14.7$ & $62.0 \pm 4.7$ & & & \\
\hline & & 60 & $1129.4 \pm 1253.8$ & $949.6 \pm 540.1$ & $345.0 \pm 9.8$ & $113.8 \pm 80.7$ & $1137.5 \pm 728.5$ & $1943.6 \pm 1161.9$ & $814.7 \pm 390.0$ & $133.7 \pm 41.1$ & & & \\
\hline \multirow{3}{*}{$\begin{array}{l}\text { 3-hydroxy-2- } \\
\text { butanone } \\
\text { (Acetoine) }\end{array}$} & \multirow{3}{*}{1291} & 1 & $71.4 \pm 27.3$ & $52.3 \pm 16.7$ & $65.5 \pm 8.7$ & $61.4 \pm 35.6$ & $23.8 \pm 0.7$ & $657.1 \pm 210.6$ & $5.2 \pm 1.9$ & $347.8 \pm 104.0$ & 0.000 & 0.000 & 0.293 \\
\hline & & 30 & $43.8 \pm 11.8$ & $56.1 \pm 35.8$ & $105.5 \pm 4.9$ & $53.4 \pm 0.6$ & $202.1 \pm 22.4$ & $244.8 \pm 47.2$ & $89.2 \pm 40.4$ & $245.7 \pm 54.8$ & & & \\
\hline & & 60 & $61.3 \pm 17.0$ & $79.4 \pm 12.4$ & $108.8 \pm 28.7$ & $24.9 \pm 34.0$ & $245.8 \pm 7.3$ & $549.0 \pm 6.4$ & $33.8 \pm 24.5$ & $256.7 \pm 54.8$ & & & \\
\hline \multirow[t]{3}{*}{ 2-Nonanon } & \multirow[t]{3}{*}{1395} & 1 & $48.9 \pm 43.1$ & $22.9 \pm 6.4$ & $20.1 \pm 9.3$ & $22.3 \pm 10.1$ & $16.6 \pm 3.0$ & $25.3 \pm 3.4$ & $18.7 \pm 0.7$ & $18.0 \pm 2.6$ & 0.161 & 0.149 & 0.000 \\
\hline & & 30 & $14.5 \pm 3.2$ & $11.5 \pm 4.2$ & $28.2 \pm 14.1$ & $14.3 \pm 2.9$ & $34.3 \pm 7.3$ & $23.1 \pm 11.8$ & $20.5 \pm 3.3$ & $14.1 \pm 0.2$ & & & \\
\hline & & 60 & $232.3 \pm 268.6$ & $161.5 \pm 119.2$ & $75.1 \pm 24.6$ & $13.4 \pm 15.1$ & $301.6 \pm 197.5$ & $565.4 \pm 486.7$ & $202.1 \pm 61.5$ & $66.7 \pm 10.8$ & & & \\
\hline \multirow{3}{*}{$\begin{array}{l}\text { 8-Nonanone- } \\
\text { 2-one }\end{array}$} & \multirow{3}{*}{1450} & 1 & $4.4 \pm 4.9$ & $1.6 \pm 0.9$ & $1.5 \pm 1.2$ & $1.1 \pm 0.0$ & $1.1 \pm 0.5$ & ND & $1.3 \pm 0.2$ & $0.4 \pm 0.0$ & 0.095 & 0.821 & 0.000 \\
\hline & & 30 & $0.7 \pm 0.3$ & $0.6 \pm 0.3$ & $3.8 \pm 0.0$ & $0.6 \pm 0.4$ & $3.0 \pm 0.7$ & ND & $1.5 \pm 0.3$ & ND & & & \\
\hline & & 60 & $46.1 \pm 55.8$ & $33.7 \pm 26.5$ & $13.1 \pm 2.4$ & $6.5 \pm 5.3$ & $54.2 \pm 44.2$ & ND & $39.5 \pm 17.9$ & ND & & & \\
\hline \multirow{3}{*}{$\begin{array}{l}\text { 2- } \\
\text { decanone }\end{array}$} & \multirow[t]{3}{*}{1501} & 1 & ND & $0.2 \pm 0.0$ & $0.2 \pm 0.0$ & ND & ND & ND & ND & ND & 0.051 & 0.150 & 0.001 \\
\hline & & 30 & $0.1 \pm 0.0$ & $0.1 \pm 0.0$ & $0.2 \pm 0.0$ & $0.1 \pm 0.0$ & $0.2 \pm 0.1$ & $0.1 \pm 0.0$ & $0.1 \pm 0.00$ & $0.1 \pm 0.0$ & & & \\
\hline & & 60 & $0.6 \pm 0.6$ & $0.3 \pm 0.2$ & ND & ND & $0.7 \pm 0.0$ & $1.4 \pm 1.2$ & $0.5 \pm 0.1$ & ND & & & \\
\hline \multirow{3}{*}{$\begin{array}{l}\text { 2- } \\
\text { undecanone }\end{array}$} & \multirow[t]{3}{*}{1607} & 1 & $5.0 \pm 3.8$ & $2.6 \pm 0.1$ & $2.4 \pm 0.6$ & $4.4 \pm 3.9$ & $2.2 \pm 0.2$ & $3.6 \pm 0.9$ & $2.6 \pm 0.3$ & $2.6 \pm 0.5$ & 0.352 & 0.166 & 0.008 \\
\hline & & 30 & $2.1 \pm 0.5$ & $1.7 \pm 0.6$ & $2.9 \pm 0.3$ & $1.8 \pm 0.2$ & $2.9 \pm 0.9$ & $2.0 \pm 0.0$ & $3.3 \pm 1.0$ & $2.3 \pm 0.1$ & & & \\
\hline & & 60 & $5.0 \pm 3.7$ & $3.5 \pm 1.4$ & $3.4 \pm 0.4$ & $2.8 \pm 1.3$ & $8.9 \pm 4.4$ & $13.2 \pm 0.1$ & $5.1 \pm 1.3$ & $2.4 \pm 0.1$ & & & \\
\hline \multicolumn{3}{|c|}{ Total ketones (11) } & 2523.3 & 1935.8 & 1374.3 & 648.2 & 3047.2 & 5549.6 & 1890.8 & 1998.5 & & & \\
\hline
\end{tabular}

RT: retention index; RM: raw material (yogurt and cream); SC: starter culture; ST: storage time (1, 30 and 60. day); YA: yogurt butter control; YB: yogurt butter produced with L. lactis subsp. lactis biovar. diacetylactis (2 \%); YC: yogurt butter produced with Leu. mesenteroides subsp. cremoris (2 \%); YD: yogurt butter produced with Leu. mesenteroides subsp. cremoris (1 \%) + L. lactis subsp. lactis biovar. diacetylactis (1 \%); CA: cream butter control; CB: cream butter produced with L. lactis subsp. lactis biovar. diacetylactis (2 \%); CC: cream butter produced with Leu. mesenteroides subsp. cremoris (2\%); CD: cream butter produced with Leu. mesenteroides subsp. cremoris $(1 \%)+$ L. lactis subsp. lactis biovar. diacetylactis (1\%)

TABLE 4. Alcohols in butters made from yogurt and cream during 60 days of storage $(\mu \mathrm{g} / 100 \mathrm{~g})$

\begin{tabular}{|c|c|c|c|c|c|c|c|c|c|c|c|c|c|}
\hline \multirow[b]{2}{*}{ Volatiles } & \multirow[b]{2}{*}{ RI } & \multirow[b]{2}{*}{ Days } & \multicolumn{8}{|c|}{ Types of butter } & \multicolumn{3}{|c|}{ Statistics, Probability } \\
\hline & & & YA & YB & YC & YD & CA & CB & CC & CD & RM & SC & ST \\
\hline \multirow[t]{3}{*}{ 2-Propanol } & \multirow[t]{3}{*}{929} & 1 & $0.8 \pm 0.0$ & $0.8 \pm 0.0$ & $1.0 \pm 0.0$ & $0.7 \pm 0.0$ & $0.5 \pm 0.0$ & ND & ND & ND & 0.000 & 0.410 & 0.000 \\
\hline & & 30 & $0.7 \pm 0.4$ & $0.5 \pm 0.5$ & $1.2 \pm 0.1$ & $0.9 \pm 0.1$ & $0.4 \pm 0.0$ & ND & $0.5 \pm 0.1$ & ND & & & \\
\hline & & 60 & $1.3 \pm 1.4$ & $0.4 \pm 0.1$ & $1.2 \pm 0.3$ & $1.3 \pm 1.0$ & $1.6 \pm 0.4$ & $0.7 \pm 0.0$ & $5.5 \pm 2.4$ & ND & & & \\
\hline \multirow[t]{3}{*}{ Ethanol } & \multirow[t]{3}{*}{934} & 1 & $3.5 \pm 0.1$ & $2.3 \pm 2.1$ & $1.8 \pm 0.6$ & $2.6 \pm 2.6$ & $65.1 \pm 3.6$ & $40.8 \pm 27.8$ & $176.0 \pm 60.6$ & $42.2 \pm 35.1$ & 0.005 & 0.000 & 0.003 \\
\hline & & 30 & $3.1 \pm 0.3$ & $1.9 \pm 0.6$ & $2.9 \pm 0.7$ & $2.1 \pm 0.3$ & $36.2 \pm 23.7$ & $27.7 \pm 9.1$ & $47.9 \pm 11.9$ & $43.5 \pm 22.7$ & & & \\
\hline & & 60 & $1.9 \pm 1.4$ & $1.7 \pm 0.5$ & $3.3 \pm 0.5$ & $4.2 \pm 3.4$ & $48.9 \pm 13.4$ & $14.7 \pm 4.5$ & $35.7 \pm 17.9$ & $34.9 \pm 25.0$ & & & \\
\hline \multirow{3}{*}{$\begin{array}{l}\text { 2-Methyl1- } \\
\text { propanol }\end{array}$} & \multirow[t]{3}{*}{1090} & 1 & ND & $0.2 \pm 0.0$ & $0.2 \pm 0.0$ & ND & $0.6 \pm 0.1$ & ND & ND & $0.6 \pm 0.0$ & 0.000 & 0.027 & 0.003 \\
\hline & & 30 & $0.1 \pm 0.0$ & $0.3 \pm 0.0$ & $0.4 \pm 0.0$ & ND & $5.4 \pm 2.1$ & ND & $0.1 \pm 0.0$ & ND & & & \\
\hline & & 60 & $0.3 \pm 0.0$ & $0.7 \pm 0.0$ & $0.6 \pm 0.9$ & $0.2 \pm 0.0$ & ND & ND & $0.8 \pm 0.0$ & ND & & & \\
\hline
\end{tabular}


TABLE 4. (Continued)

\begin{tabular}{|c|c|c|c|c|c|c|c|c|c|c|c|c|c|}
\hline \multirow[b]{2}{*}{ Volatiles } & \multirow[b]{2}{*}{ RI } & \multirow[b]{2}{*}{ Days } & \multicolumn{8}{|c|}{ Types of butter } & \multicolumn{3}{|c|}{ Statistics, Probability } \\
\hline & & & YA & YB & YC & YD & CA & CB & CC & $\mathrm{CD}$ & RM & SC & ST \\
\hline \multirow[t]{3}{*}{ 2-Pentanol } & \multirow[t]{3}{*}{1119} & 1 & $2.2 \pm 0.0$ & $0.1 \pm 0.0$ & $1.3 \pm 0.0$ & ND & $0.0 \pm 0.0$ & $0.0 \pm 0.0$ & $0.2 \pm 0.6$ & $0.1 \pm 0.0$ & 0.005 & 0.002 & 0.000 \\
\hline & & 30 & ND & ND & ND & ND & $1.0 \pm 0.4$ & ND & $1.6 \pm 0.4$ & $0.1 \pm 0.1$ & & & \\
\hline & & 60 & $2.8 \pm 3.4$ & $4.3 \pm 4.5$ & $0.8 \pm 0.5$ & ND & $7.6 \pm 3.9$ & $39.9 \pm 38.1$ & $77.0 \pm 51.7$ & $0.4 \pm 0.2$ & & & \\
\hline \multirow[t]{3}{*}{ 1-Butanol } & \multirow[t]{3}{*}{1144} & 1 & $0.4 \pm 0.1$ & $0.5 \pm 0.0$ & $0.5 \pm 0.0$ & $0.4 \pm 0.0$ & $4.7 \pm 0.5$ & $1.0 \pm 0.2$ & $0.7 \pm 0.3$ & $0.6 \pm 0.0$ & 0.000 & 0.000 & 0.000 \\
\hline & & 30 & $0.5 \pm 0.2$ & $0.5 \pm 0.2$ & $0.7 \pm 0.1$ & $0.5 \pm 0.2$ & $102.8 \pm 71.0$ & $1.2 \pm 0.7$ & $1.1 \pm 1.2$ & $0.8 \pm 0.0$ & & & \\
\hline & & 60 & $1.4 \pm 1.3$ & $0.4 \pm 0.3$ & $0.7 \pm 0.4$ & $0.9 \pm 0.8$ & $477.0 \pm 0.0$ & $4.6 \pm 1.3$ & ND & $0.7 \pm 0.1$ & & & \\
\hline \multirow{3}{*}{$\begin{array}{l}\text { 1-Pentene- } \\
\text { 3-ol }\end{array}$} & \multirow{3}{*}{1158} & 1 & ND & ND & ND & ND & ND & $0.4 \pm 0.1$ & ND & $0.2 \pm 0.0$ & 0.000 & 0.000 & 0.008 \\
\hline & & 30 & ND & ND & ND & ND & $0.3 \pm 0.0$ & $0.4 \pm 0.0$ & ND & $0.7 \pm 0.1$ & & & \\
\hline & & 60 & $0.2 \pm 0.2$ & $0.1 \pm 0.0$ & $0.2 \pm 0.0$ & $0.2 \pm 0.0$ & ND & $0.8 \pm 0.6$ & ND & $0.6 \pm 0.3$ & & & \\
\hline \multirow{3}{*}{$\begin{array}{l}\text { 3-methy-1- } \\
\text { butanol }\end{array}$} & \multirow{3}{*}{1207} & 1 & $2.4 \pm 1.2$ & $1.2 \pm 0.1$ & $1.9 \pm 0.1$ & $1.6 \pm 0.9$ & $1.4 \pm 0.5$ & $0.9 \pm 0.5$ & $0.8 \pm 0.0$ & $0.8 \pm 0.6$ & 0.000 & 0.000 & 0.000 \\
\hline & & 30 & $2.2 \pm 0.3$ & $1.7 \pm 0.7$ & $2.5 \pm 0.3$ & $1.2 \pm 0.5$ & $77.8 \pm 22.0$ & $0.9 \pm 0.4$ & $4.8 \pm 1.6$ & $0.9 \pm 0.5$ & & & \\
\hline & & 60 & $3.3 \pm 2.1$ & $3.1 \pm 0.2$ & $3.6 \pm 1.7$ & $1.4 \pm 0.7$ & $101.6 \pm 26.3$ & $1.3 \pm 1.2$ & $7.3 \pm 1.6$ & $0.4 \pm 0.0$ & & & \\
\hline \multirow[t]{3}{*}{ 1-pentanol } & \multirow[t]{3}{*}{1250} & 1 & $3.1 \pm 0.3$ & $1.6 \pm 0.3$ & $2.1 \pm 0.3$ & $1.4 \pm 0.8$ & $1.7 \pm 0.2$ & $3.7 \pm 0.2$ & $1.9 \pm 0.5$ & $3.0 \pm 0.1$ & 0.000 & 0.000 & 0.000 \\
\hline & & 30 & $2.4 \pm 0.5$ & $1.6 \pm 0.7$ & $2.5 \pm 0.5$ & $1.8 \pm 0.1$ & $5.6 \pm 2.7$ & $5.8 \pm 2.3$ & $1.3 \pm 0.1$ & $4.5 \pm 0.1$ & & & \\
\hline & & 60 & $3.5 \pm 1.0$ & $3.9 \pm 0.4$ & $2.8 \pm 0.5$ & $1.3 \pm 0.5$ & $33.4 \pm 10.6$ & $6.2 \pm 0.5$ & $0.6 \pm 0.2$ & $4.0 \pm 0.6$ & & & \\
\hline \multirow[t]{3}{*}{ 2-Heptanol } & \multirow[t]{3}{*}{1319} & 1 & ND & ND & ND & ND & ND & $0.3 \pm 0.0$ & ND & $0.7 \pm 0.1$ & 0.241 & 0.064 & 0.084 \\
\hline & & 30 & ND & ND & $0.5 \pm 0.0$ & ND & ND & $0.4 \pm 0.2$ & $4.4 \pm 1.2$ & $0.4 \pm 0.3$ & & & \\
\hline & & 60 & $1.2 \pm 0.0$ & $0.9 \pm 0.3$ & $0.5 \pm 0.2$ & ND & $3.08 \pm 0.8$ & $16.2 \pm 16.5$ & ND & $0.5 \pm 0.2$ & & & \\
\hline \multirow{3}{*}{$\begin{array}{l}\text { 3-methyl- } \\
\text { 2-buten- } \\
\text { 1-ol }\end{array}$} & \multirow[t]{3}{*}{1322} & 1 & ND & ND & $1.4 \pm 0.1$ & $0.5 \pm 0.2$ & ND & ND & ND & $0.5 \pm 0.0$ & 0.002 & 0.000 & 0.501 \\
\hline & & 30 & $0.5 \pm 0.3$ & $0.2 \pm 0.1$ & $0.3 \pm 0.4$ & $0.2 \pm 0.1$ & ND & ND & ND & $0.2 \pm 0.0$ & & & \\
\hline & & 60 & $1.0 \pm 0.0$ & $0.2 \pm 0.1$ & $0.5 \pm 0.2$ & $0.2 \pm 0.0$ & ND & ND & ND & ND & & & \\
\hline \multirow[t]{3}{*}{ 1-Hexanol } & \multirow[t]{3}{*}{1351} & 1 & $3.5 \pm 1.4$ & $2.1 \pm 0.6$ & $2.7 \pm 0.2$ & $1.7 \pm 0.5$ & $1.2 \pm 0.3$ & $1.4 \pm 0.0$ & $0.9 \pm 0.6$ & $1.0 \pm 0.1$ & 0.000 & 0.000 & 0.000 \\
\hline & & 30 & ND & & $5.1 \pm 1.4$ & ND & $93.1 \pm 74.0$ & $7.2 \pm 3.0$ & $4.0 \pm 0.7$ & $3.2 \pm 0.0$ & & & \\
\hline & & 60 & ND & $5.8 \pm 0.2$ & $4.6 \pm 0.5$ & ND & $562.9 \pm 0.0$ & $73.0 \pm 2.9$ & $8.9 \pm 3.4$ & $4.8 \pm 1.3$ & & & \\
\hline 1-heptanol & 1456 & 1 & $0.5 \pm 0.3$ & $0.2 \pm 0.0$ & $0.8 \pm 0.4$ & $0.2 \pm 0.2$ & $0.4 \pm 0.0$ & $9.0 \pm 0.0$ & $0.4 \pm 0.0$ & ND & 0.000 & 0.000 & 0.000 \\
\hline & & 30 & $0.5 \pm 0.0$ & $0.3 \pm 0.0$ & $0.6 \pm 0.1$ & $0.3 \pm 0.1$ & ND & ND & $0.9 \pm 0.2$ & ND & & & \\
\hline & & 60 & $1.2 \pm 0.7$ & $1.0 \pm 0.2$ & $0.8 \pm 0.2$ & $0.6 \pm 0.3$ & ND & ND & ND & ND & & & \\
\hline 2-ethyl-1- & 1490 & 1 & $0.9 \pm 0.1$ & $0.6 \pm 0.3$ & $0.8 \pm 0.4$ & $0.7 \pm 0.5$ & $0.2 \pm 0.0$ & $0.3 \pm 0.0$ & $0.3 \pm 0.0$ & $0.2 \pm 0.0$ & 0.028 & 0.002 & 0.106 \\
\hline hexanol & & 30 & $0.6 \pm 0.1$ & $0.4 \pm 0.1$ & $1.3 \pm 1.2$ & $0.5 \pm 0.2$ & $0.6 \pm 0.2$ & $0.6 \pm 0.2$ & $1.4 \pm 0.3$ & $0.4 \pm 0.0$ & & & \\
\hline & & 60 & $0.9 \pm 0.0$ & $0.7 \pm 0.1$ & $1.1 \pm 0.6$ & $0.4 \pm 0.4$ & $0.5 \pm 0.1$ & $0.3 \pm 0.1$ & ND & $0.2 \pm 0.1$ & & & \\
\hline 2-nonanol & 1519 & 1 & $3.7 \pm 4.30$ & $1.4 \pm 0.5$ & $1.3 \pm 0.9$ & $1.2 \pm 0.4$ & $1.0 \pm 0.3$ & $1.3 \pm 0.2$ & $0.7 \pm 0.0$ & $0.7 \pm 0.0$ & 0.591 & 0.332 & 0.014 \\
\hline & & 30 & $0.5 \pm 0.1$ & $0.4 \pm 0.3$ & $0.7 \pm 0.0$ & $0.4 \pm 0.1$ & $0.3 \pm .00$ & $0.4 \pm 0.1$ & $0.4 \pm 0.2$ & $0.4 \pm 0.1$ & & & \\
\hline & & 60 & $0.4 \pm 0.5$ & $0.4 \pm 0.2$ & $0.3 \pm 0.1$ & $0.3 \pm 0.2$ & ND & $1.6 \pm 1.7$ & $0.7 \pm 0.1$ & ND & & & \\
\hline 1-octanol & 1560 & 1 & $0.4 \pm 0.0$ & $0.4 \pm 0.1$ & $0.3 \pm 0.0$ & $0.2 \pm 0.1$ & $0.2 \pm 0.0$ & $0.3 \pm 0.0$ & $0.2 \pm 0.1$ & $0.3 \pm 0.1$ & 0.000 & 0.000 & 0.000 \\
\hline & & 30 & $0.3 \pm 0.0$ & $0.4 \pm 0.1$ & $0.4 \pm 0.1$ & $0.2 \pm 0.0$ & $3.4 \pm 2.0$ & $0.5 \pm 0.0$ & $1.2 \pm 0.2$ & $1.1 \pm 0.7$ & & & \\
\hline & & 60 & $0.8 \pm 0.6$ & $0.6 \pm 0.2$ & $0.5 \pm 0.2$ & $0.4 \pm 0.2$ & $70.4 \pm 0.4$ & $15.2 \pm 4.7$ & $4.1 \pm 2.2$ & $2.1 \pm 1.1$ & & & \\
\hline & 1581 & 1 & $1.0 \pm 0.0$ & $2.2 \pm 0.0$ & $0.6 \pm 0.0$ & $0.9 \pm 0.0$ & ND & ND & ND & $4.0 \pm 0.9$ & 0.001 & 0.000 & 0.000 \\
\hline butanodiol & & 30 & $0.4 \pm 0.4$ & $0.5 \pm 0.0$ & ND & ND & $17.8 \pm 0.0$ & $1.2 \pm 0.0$ & $15.8 \pm 8.3$ & $25.8 \pm 5.4$ & & & \\
\hline & & 60 & ND & ND & ND & ND & $27.0 \pm 0.0$ & $12.9 \pm 0.0$ & $48.2 \pm 22.6$ & $41.9 \pm 2.4$ & & & \\
\hline Total alcoho & ol (16) & & 54.4 & 46.5 & 57.3 & 31.6 & 1756.8 & 293.1 & 456.3 & 227.4 & & & \\
\hline
\end{tabular}

RT: retention index; RM: raw material (yogurt and cream); SC: starter culture; ST: storage time (1, 30 and 60. day); YA: yogurt butter control; YB: yogurt butter produced with L. lactis subsp. lactis biovar. diacetylactis (2 \%); YC: yogurt butter produced with Leu. mesenteroides subsp. cremoris (2 \%); YD: yogurt butter produced with Leu. mesenteroides subsp. cremoris (1 \%) + L. lactis subsp. lactis biovar. diacetylactis (1 \%); CA: cream butter control; CB: cream butter produced with L. lactis subsp. lactis biovar. diacetylactis (2\%); CC: cream butter produced with Leu. mesenteroides subsp. cremoris (2 \%); CD: cream butter produced with Leu. mesenteroides subsp. cremoris (1\%) + L. lactis subsp. lactis biovar. diacetylactis (1\%) 
TABLE 5. Carboxylic acids in butters made from yogurt and cream during 60 days of storage $(\mu \mathrm{g} / 100 \mathrm{~g})$

\begin{tabular}{|c|c|c|c|c|c|c|c|c|c|c|c|c|c|}
\hline \multirow[b]{2}{*}{ Volatiles } & \multirow[b]{2}{*}{ RI } & \multirow[b]{2}{*}{ Days } & \multicolumn{8}{|c|}{ Types of butter } & \multicolumn{3}{|c|}{ Statistics, Probability } \\
\hline & & & YA & YB & YC & YD & CA & CB & CC & CD & RM & SC & ST \\
\hline \multirow{3}{*}{$\begin{array}{l}\text { Acetic } \\
\text { acid }\end{array}$} & \multirow[t]{3}{*}{1469} & 1 & $13.7 \pm 2.7$ & $30.9 \pm 34.7$ & $19.6 \pm 7.9$ & $23.5 \pm 18.9$ & $11.6 \pm 11.8$ & $241.7 \pm 138.2$ & $40.4 \pm 32.9$ & $169.1 \pm 95.1$ & 0.000 & 0.000 & 0.033 \\
\hline & & 30 & $18.2 \pm 4.4$ & $34.1 \pm 22.4$ & $32.3 \pm 8.8$ & $16.2 \pm 2.3$ & $107.7 \pm 8.2$ & $215.8 \pm 100.8$ & $16.4 \pm 1.7$ & $396.8 \pm 25.1$ & & & \\
\hline & & 60 & $14.7 \pm 3.4$ & $23.7 \pm 22.2$ & $29.9 \pm 1.9$ & $26.8 \pm 21.8$ & $138.0 \pm 50.5$ & $382.4 \pm 233.5$ & $67.2 \pm 22.3$ & $397.1 \pm 78.3$ & & & \\
\hline \multirow{3}{*}{$\begin{array}{l}\text { 2-Methyl- } \\
\text { propanoic } \\
\text { acid }\end{array}$} & \multirow[t]{3}{*}{1588} & 1 & ND & ND & ND & ND & ND & ND & ND & ND & 0.000 & 0.000 & 0.000 \\
\hline & & 30 & $0.4 \pm 0.0$ & $1.6 \pm 1.7$ & ND & ND & $8.3 \pm 0.0$ & $3.9 \pm 4.4$ & ND & ND & & & \\
\hline & & 60 & $3.1 \pm 0.0$ & $0.6 \pm 0.3$ & $0.9 \pm 0.0$ & $0.6 \pm 0.0$ & $10.1 \pm 0.0$ & ND & ND & ND & & & \\
\hline \multirow{3}{*}{$\begin{array}{l}\text { Butanoic } \\
\text { (butyric) } \\
\text { acid }\end{array}$} & \multirow[t]{3}{*}{1650} & 1 & $39.3 \pm 22.6$ & $37.6 \pm 33.8$ & $26.2 \pm 10.3$ & $14.1 \pm 26.2$ & $26.2 \pm 11.1$ & $100.9 \pm 53.7$ & $17.0 \pm 19.5$ & $76.3 \pm 28.7$ & 0.005 & 0.001 & 0.001 \\
\hline & & 30 & $72.4 \pm 10.3$ & $53.6 \pm 16.0$ & $65.6 \pm 9.2$ & $21.0 \pm 4.2$ & $936.4 \pm 354.5$ & $129.3 \pm 8.7$ & $20.7 \pm 8.8$ & $134.2 \pm 3.2$ & & & \\
\hline & & 60 & $267.5 \pm 132.4$ & $156.7 \pm 142.1$ & $75.8 \pm 23.8$ & $64.0 \pm 31.2$ & $2074.7 \pm 666.6$ & $297.0 \pm 139.9$ & $454.6 \pm 299.7$ & $136.2 \pm 21.7$ & & & \\
\hline \multirow{3}{*}{$\begin{array}{l}\text { 3-methyl- } \\
\text { butanoic } \\
\text { acid }\end{array}$} & \multirow[t]{3}{*}{1700} & 1 & $1.8 \pm 1.4$ & $1.4 \pm 0.8$ & $1.1 \pm 0.0$ & $1.4 \pm 0.9$ & ND & ND & ND & ND & 0.000 & 0.000 & 0.000 \\
\hline & & 30 & $1.9 \pm 0.0$ & $1.7 \pm 0.7$ & $2.5 \pm 0.3$ & $0.8 \pm 0.0$ & $8.2 \pm 3.5$ & $2.4 \pm 0.1$ & ND & $1.3 \pm 0.0$ & & & \\
\hline & & 60 & $2.9 \pm 2.1$ & $1.6 \pm 0.0$ & $1.9 \pm 0.5$ & $1.3 \pm 1.0$ & $12.7 \pm 2.2$ & $6.7 \pm 4.6$ & $4.1 \pm 0.0$ & ND & & & \\
\hline \multirow{3}{*}{$\begin{array}{l}\text { Pentanoic } \\
\text { acid }\end{array}$} & \multirow{3}{*}{1766} & 1 & ND & $0.8 \pm 0.0$ & ND & ND & ND & ND & ND & ND & 0.000 & 0.000 & 0.000 \\
\hline & & 30 & ND & ND & ND & ND & $4.1 \pm 0.0$ & ND & ND & ND & & & \\
\hline & & 60 & $0.5 \pm 0.7$ & ND & ND & ND & $5.7 \pm 0.7$ & ND & ND & ND & & & \\
\hline \multirow{3}{*}{$\begin{array}{l}\text { Hexanoic } \\
\text { acid }\end{array}$} & \multirow{3}{*}{$\begin{array}{l}1870 \\
(1861)\end{array}$} & 1 & $81.2 \pm 31.8$ & $43.8 \pm 21.2$ & $42.5 \pm 1.3$ & $51.8 \pm 41.9$ & $33.4 \pm 4.0$ & $31.1 \pm 9.8$ & $34.8 \pm 11.1$ & $47.3 \pm 6.2$ & 0.000 & 0.010 & 0.000 \\
\hline & & 30 & $69.1 \pm 9.3$ & $59.3 \pm 21.3$ & $80.2 \pm 13.6$ & $39.9 \pm 3.2$ & $205.8 \pm 70.4$ & $40.7 \pm 5.9$ & $31.4 \pm 9.6$ & $62.0 \pm 2.7$ & & & \\
\hline & & 60 & $100.6 \pm 9.1$ & $66.8 \pm 15.8$ & $59.3 \pm 11.9$ & $53.0 \pm 14.3$ & $447.4 \pm 158.3$ & $204.0 \pm 162.1$ & $57.6 \pm 25.5$ & $40.7 \pm 0.1$ & & & \\
\hline \multicolumn{2}{|c|}{ Total acids (6) } & & 687.3 & 514.2 & 437.8 & 314.4 & 4030.3 & 1655.9 & 744.2 & 1461.0 & & & \\
\hline
\end{tabular}

RT: retention index; RM: raw material (yogurt and cream); SC: starter culture; ST: storage time (1, 30 and 60. day); YA: yogurt butter control; YB: yogurt butter produced with L. lactis subsp. lactis biovar. diacetylactis (2\%); YC: yogurt butter produced with Leu. mesenteroides subsp. cremoris (2 \%); YD: yogurt butter produced with Leu. mesenteroides subsp. cremoris (1 \%) + L. lactis subsp. lactis biovar. diacetylactis (1 \%); CA: cream butter control; CB: cream butter produced with L. lactis subsp. lactis biovar. diacetylactis (2 \%); CC: cream butter produced with Leu. mesenteroides subsp. cremoris (2\%); CD: cream butter produced with Leu. mesenteroides subsp. cremoris $(1 \%)+$ L. lactis subsp. lactis biovar. diacetylactis (1\%) 
Esters, which give a fruity flavour, are one of most abundant volatiles in dairy products and low concentrations of esters are contribute to the flavour balance in food (Puniya, 2016). In general, higher concentrations of ester compounds were determined in cream butter than in yogurt samples. All factors (raw material, starter type, and storage period) had statistically affected all types of esters compounds. However, starter type and storage period were not statistically significant for the formation of methyl butanoate, methyl decanoate and methyl dodecanoate (Table 2). Ethyl acetate, ethyl butanoate, ethyl octanoate, methyl decanoate, and methyl dodecanoate were determined during storage in both butter samples. S-methyl thioacetate was only observed in yogurt butter samples during storage. Thioesters contain a free sulphydryl group that gives the characteristic aroma of onion, garlic and some fruits (Yahia, 2017). It was thought that thioester in yogurt butter samples may be formed by a thermal process, i.e., during heat treatment of yogurt milk (Yu and Ho, 1995). The starter and storage period had significant effects on the esters $(P<0.01)$. While ethyl acetate was lower in YB and $C B$ samples produced with $L$. lactis subsp. lactis biovar. diacetylactis at the end of storage, ethyl butanoate, ethyl octanoate, methyl deconoate and methyl dodeconate were lower in YD and CD samples produced with the mixture of Leu. mesenteroides subsp. cremoris and L. lactis subsp. lactis biovar. diacetylactis at the end of storage. It was reported that these esters depende on the type of microorganism, environmental conditions, and precursors for the formation of esters (Ruiz Rodríguez et al., 2019). The data from GC-MS analysis were subjected to PCA which was a good tool to reduce large data to understand any differences between the samples (Figure 1).

Principal components PC1 and PC2 contained $30 \%$ and $18 \%$ of the total variance, respectively (Figure 1). From the PCA analysis, yogurt and cream butter samples were separated on the plot. While yogurt butter samples were located on the positive side of $\mathrm{PC} 1$, cream butter samples were positioned on the negative side of the same component. Although all cream butter samples were located in the same group, 60 day-old cream butter

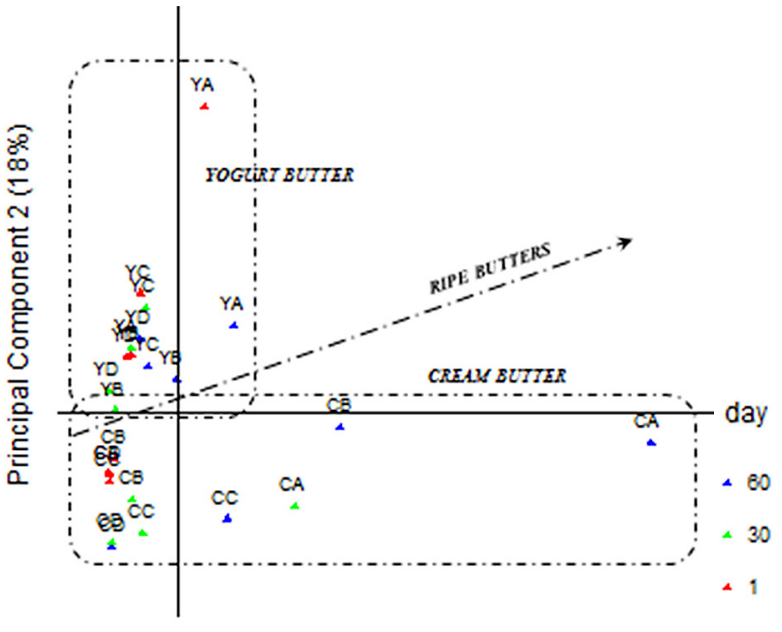

Principal Component $1(30 \%)$

YA: yogurt butter control; YB: yogurt butter produced with L. lactis subsp. lactis biovar. diacetylactis (2\%); YC: yogurt butter produced with Leu. mesenteroides subsp. cremoris (2 $\%)$; Y: yogurt butter produced with Leu. mesenteroides subsp. cremoris (1 \%) + L. lactis subsp. lactis biovar. diacetylactis (\% 1); CA: cream butter control; CB: cream butter produced with $L$. lactis subsp. lactis biovar. diacetylactis (2 \%); CC: cream butter produced with Leu. mesenteroides subsp. cremoris (2\%); CD: cream butter produced with Leu. mesenteroides subsp. cremoris $(1 \%)+$ L. lactis subsp. lactis biovar. diacetylactis (1\%), lower letters in a row (same samples in different storage days) and capital letters (different samples in same storage days) with no common superscript differ $(\mathrm{P}<0.05)$.

FIGURE 1. Principal component analysis (PCA) of the volatiles data of butter samples during 1, 30 and 60 days of storage

exhibited a different volatile profile, as shown in the plot. This butter sample contained high levels of 2-pentanone, methyl butanoate, 3-methyl-botanoate, hexanal, 3-methyl-1-butanol, butyric acid, and hexanoic acid. On the other hand, for all samples of yogurt butter grouped together, the storage period or ripening conditions only had a slight effect on the volatile profile of yogurt samples, as shown on the plot. This can be explained by the suppression of yogurt starters on Leu. mesenteroides subsp. cremoris and L. lactis subsp. lactis biovar. diacetylactis, which did not grow as much as yogurt starters (not data shown). However, use of the starter in cream butter showed predominant activity in the butter samples, resulting in less hexanoic and butyric acid production, which were found at high levels in control cream butter. 


\section{Aldehydes and ketones}

The total concentration of aldehydes was higher in cream butter than in yogurt samples. Additionally, 3-methyl-2-butanal, 3-methyl butanal, and butanal were determined at different storage periods in both types of butter, and they had fluctuating levels. 3-methyl-2-butanal was not affected by the raw material; however, benzaldehyde (by starter type), 3-methyl butanal (by storage time), butanal (by starter culture and storage time) and acetaldehyde (by raw material and storage time) were not affected by the factors in the parenthesis (Table 3). As seen in Table 3, acetaldehyde and benzaldehyde were the most abundant aldehydes determined in butter samples. The level of acetaldehyde decreased in yogurt butter; while its concentration increased in cream butter samples at the end of storage period. Although acetaldehyde was highest in the YA sample on the first day, it was also high in the YD and CB samples of butter at the end of storage. Decrease in the acetaldehyde and ethanol levels in yogurt butter samples coincided with an increasing acetic acid level (Tables 3, 4 and 5). Gürkan and Hayaloglu (2017) also found that the amount of acetaldehyde decreased in yoghurt during storage. Some authors reported that the conversion of acetaldehyde, acetic acid and ethanol was correlated with water activity and pH (Liu et al., 1997). In the present study, the $\mathrm{pH}$ of yogurt butter samples was lower when compared to those of cream butter. The acidity levels were vice versa. The benzaldehyde level was increased in both butter types throughout the storage period and there were some fluctuations. The lowest benzaldehyde level was detected in the CD sample and the highest was found in the control sample in yogurt and cream butters at the end of storage. The starter culture had a significant effect on acetaldehyde at a $\mathrm{P}<0.01$ level. The level of hexanal was higher in cream butter.

Methyl ketones are normally principal flavour compounds in Blue cheeses and arise by the enzymatic oxidation of free fatty acids in dairy products (Engels et al., 1997). Some bacteria are able to form methyl ketones via the oxidation of aliphatic hydrocarbons at the methylene carbon a to the methyl group (McSweeney and Sousa, 2000). On the other hand, cheeses, milk, butter, and other dairy products have been shown contain these compounds in addition to the plants and their products
(Kaminarides et al., 2007). In this study, a total of 11 ketones, the most abundant of which were 2-pentanone, 2- heptanone, acetoin and 2-nonanone, were found in both butter types (Table 3). Starter type did not change the concentration of 2-propanone, 2-heptanone. On the other hand, acetoine was influenced by starter type, while the levels of 2-nonanon, 8-nonanone-2-one and 2-undecanone was not changed by raw material and starter type (Table 3 ). Generally, ketone concentrations were high in B samples of butter. The starter culture had no significant effect on ketones except for 2-pentanone, 2,3-pentandione, 2-butanone and diacetyl, but the storage time was generally important $(P<0.01)$. Although the total ketone levels were high in yogurt control sample, the B sample had the highest concentration compared to the $C$ and $D$ samples. These could be due to the differences in combination of starter bacteria and their growth conditions in yogurt or cream. Diacetyl is one of dominant carbonyl compound in butter, yogurt and other milk products (Clark and Winter, 2015; Demirkol et al., 2016). The diacetyl level was highest in YD and CD samples, which were produced with mixed culture. In general, the levels of diacetyl decreased in all samples with exception of YD and CD samples throughout the storage period. In addition, diacetyl was disappeared in the CA and CC samples at the end of storage. These results showed that mixed starter cultures formed more diacetyl than the single ones. Edris and Schirle-Keller (2010) reported that although diacetyl is generally produced by strains of Leuconostoc, it is also produced in high levels by $\mathrm{S}$. diacetylactis, Leu. citrovorum and some strains of Leu. dextranicum. Lactococcus lactis subsp. lactis biovar. diacetylactis which was isolated from a naturally fermented milk. The latter one was also found to produce malt-flavour in milk products in addition to other aroma compounds such as diacetyl, acetoin, and acetaldehyde (Mutukumira, 1996). Same authors noted that citrate negative Lactococcus lactis subsp. lactis produce undesirable malty compounds in dairy products. Reineccius (2006) reported that diacetyl produced by starter culture is not stable enough because it converts into acetoin and 2,3-butanodiol via diacetyl reductase enzyme of the microorganism. Generally, the ketones detected in cream butter samples in the present study were also detected in other studies, such as Peterson and Reineccius (2003) or Lozano et al. (2007). 
Alcohols

In comparison to other compounds alcohols contribute more or less to the flavour when they are not saturated (1-octen-3-ol) or at a high level (Reineccius, 2006). As seen from Table 4, total alcohols were considerably higher in cream butter samples than in yoghurt samples. 1-pentanol, 3-methyl-1-butanol, 1-octanol, 1-butanol were detected in increasing levels in all butter samples throughout the storage period. The lowest alcohols were determined in samples of $D$ for the both types of butter. Type of starter culture did not influence the level of 2-propanol and 2-nonanol. Also, storage time did not change the level of 3-methyl-2-buten-1-ol, 2-ethyl-1-hexanol and 2-heptanol. The concentrations of alcohols and acids significantly changed in relation to the type of raw material, starter culture and storage time (Tables 4 and 5).

The level of ethanol was higher in cream butter samples than in yogurt samples and it showed some fluctuations during storage. In some sampling times, the levels of ethanol were also increased in the YC and YD samples containing Leuconostoc sp.; but not higher than those in cream butter samples. Such results can be explained by the combined effect of yogurt and butter cultures. Its decrease in cream butter samples could be due to the decreasing number of Leu. mesenteroides subsp. cremoris (data not shown). Additionally, Karatapanis et al. (2006) reported that the packaging material affected the ethanol level and that excessive ethanol formed in polyethylene and carton packaging material because of ethyl and methyl esters of butyric and hexanoic acid as well as methyl esters of octanoic acid. Additionally, Lb. delbrueckii subsp. bulgaricus and S. thermophilus, which have aldehyde and alcohol dehydrogenase enzyme, can catalyse glucose and form acetaldehyde and ethanol (Tamime and Robinson, 2007).

\section{Carboxylic acids}

In this study acetic acid, 2-methyl-propionic acid, 3-methyl-butanoic acid, pentanoic acid, butyric acid and hexanoic acid were among the detected volatile acid compounds (Table 5). Starter culture, raw material and storage time were statistically important for all acids $(P<0.01)$. The total carboxylic acids were considerably higher in cream butter samples than in yogurt butter samples. Acetic, butyric and hexanoic acid were present duirng the entire storage period in both butter types, and butyric acid was the highest. Demirkol et al. (2006) found acetic (15.67-62642 $\mu \mathrm{g} / \mathrm{kg})$ and butyric acid $(1113-89605 \mu \mathrm{g} / \mathrm{kg})$ in butter samples provided from local market. Butyric acid is produced by the fermentation of butyric acid bacteria or degradation of amino acids, and the other volatile fatty acids are formed from lactate (Fox and Wallace, 1997). Butyric acid increased in all butter samples throughout the storage time. The lowest butyric acid level was observed in sample $D$ and the highest was seen in the control butter samples at the end of storage. There are different results in the literature for butyric acid. For example, while Ozcan et al. (2016) determined butyric acid present in Trabzon butters between 1.81 and 2.17, Karaca et al. (2018) found that butyric acid content of control and kefir cultured butter samples were 0.698 and 0.337 $\%$, respectively. Beshkova et al. (1998) found 8.9$10.8 \mathrm{mg} / \mathrm{kg}$ for yogurt samples. The concentration of free acids, including butyric acid, changed by lipolysis and the action of membrane lipases or native lipases present in milk during the manufacture of yogurt or cream before butter-making. The other important result was that although hexanoic acid increased at the end of the storage period compared to the first day, there were some fluctuations at intermediate storage times in all butter samples, except in the sample CD, which decreased at the end of storage. The lowest level of this acid was formed in the sample $D$ in both butter sample types at the end of storage. Nájera-Domínguez et al. (2014) reported that the origin of Lactotoccus lactis (vegetables, raw milk products, and industrial cultures) can be a reason for variation in volatile compounds although some compounds were same such as acetic, lactic, butyric, and caproic acids, acetoin, 3-methyl-1-butanol, and 2,3-butanediol. On the other hand, these authors noted that the metabolic and genetic differences of these strains were not clear and more research is required to understand these. Narvhus et al. (1998) showed that incubation temperature had a significant effect on growth rate of starter and the rate of production and amount of the malty-like compounds and lactate, diacetyl, ethanol, acetoin and acetaldehyde. On the other hand, same authors reported that adaptation of $L$. lactis from plant environment to the milk environment could cause the loss of certain metabolic 
capabilities derived. L. lactis isolated from vegetables or raw milk products are capable of generate flavors different from those produced by industrial strains (Ayad et al., 2001; López-González et al., 2018).

\section{Other compounds}

In this study, two sulphides, three terpenes and three miscellanous compounds were identified in both butter types. Two sulphide compounds, including carbon disulphide and dimethyl disulphide were detected. Sulphur compounds were higher in yogurt butter samples than in cream samples. This could be due to the protein content and the high heat treatment of yogurt milk. The highest level of carbon disulphide was observed in YA and CC samples. It was reported that the formation of dimethyl disulfide and dimethyl trisulfide can be a result of further oxidation of methanethiol formed by strecker degradation of methionine (Kaminadires et.al., 2013). Terpene compounds originate from plants and can be found in milk and milk products depending on the feeding conditions (Valdivielso et al. 2017). The levels of terpenes, especially of a-pinene, $\beta$-pinen, and champane were higher in yogurt butter than cream, which was probably due to the processing of milk obtained from the grazing in pasture. The miscellanous volatiles were chloroform, toluene and styrene. Toluene and styrene were also found in Erzincan Tulum cheese samples during ripening (Çakir et al., 2016). Some researchers noted that these compound types can be detected on roads, soils, and in and out of barns (Ciganek and Neca, 2008). Lozano et al. (2007) showed that the packaging material had an important effect on the styrene level in butter samples packed with aluminium foil and wax material as well as reported that aluminium foil was better for inhibiting the passage of styrene to the product. On the other hand, toluene could result from hydrolysis of $\beta$-carotene, while chloroform could be from sanitizing materials used for milking equipment (Marsili and Miller, 1998).

\section{Sensory properties}

As seen from Table 6, the sensory attributes of the butter samples after 1, 15, 30, 45 and 60 days of storage were evaluated. All samples were given good scores by the assessors. However, butter samples produced with starter cultures generally had higher scores than control samples at the end of storage. All butter samples showed a decreasing tendency towards the end of the storage period, and control samples of both butter types had the lowest scores. There was no difference in the colour, texture, and odour scores between yogurt and cream butter samples at the end of storage. On the first day of storage, the YB sample was significantly different for the flavour, rancid taste and general acceptability scores compared to other samples, but there was no difference at the end of storage. However, the highest flavour score was given to the YB sample at the end of storage. This could be due to the acetaldehyde and diacetyl production by $L$. lactis subsp. lactis biovar. diacetylactis, which is responsible for the butter aroma. On the other hand, the highest rancid taste and general acceptability were given to the YD sample at the end of storage. The rancid taste scores decreased as the storage period proceeded. According to the sensory evaluation test, the scores between 9 to 8; 7-6; 5-4; and 3-2 indicated 'no rancid taste'; 'little rancid taste'; 'perceived rancid taste' and 'cannot be accepted' respectively. The observed decrease in rancid taste scores was most probably caused by the increase in free fatty acids during the shelf-life especially butyric acids as seen in Table 5. Rancid flavour and odour appeared most probably due to the lipolysis of milk fat. The short-chain free acids (especially C4:0 and (6:0) from milk fats hydrolysed by lipase enzyme which can be of natural and of microbial origin, are responsible for rancid character (Couvreur et al., 2006). Also the values for peroxide in the butters changed from 0.41 to 0.94 meq $\mathrm{O}_{2} / \mathrm{kg}$ milk fat (data not shown) which were tolerable values for butter samples in Turkey. Although all properties were close to each other during the storage period, butter samples produced with starter culture were more preferred than the control ones. The sample D containing mixture of starters had the highest scores for general acceptability at the end of storage. Based on the result of the sensory scores, butter produced with starter had higher scores than control samples at the end of storage. Karaca et al. (2018) reported that butters produced with natural kefir culture had better sensory properties than 
TABLE 6. Sensory analysis of butters made from yogurt and cream during 60 days of storage

\begin{tabular}{|c|c|c|c|c|c|c|c|c|c|}
\hline \multirow[b]{2}{*}{ Variants } & \multirow[b]{2}{*}{ Days } & \multicolumn{8}{|c|}{ Types of butter } \\
\hline & & YA & YB & YC & YD & CA & CB & CC & $C D$ \\
\hline \multirow[t]{5}{*}{ Colour } & 1 & $8.1 \pm 0.1^{\mathrm{BC} . \mathrm{b}}$ & $8.3 \pm 0 .{ }^{18 c . c}$ & $8.2 \pm 0.0^{B C . a}$ & $7.9 \pm 0.1^{\text {AB.ab }}$ & $7.9 \pm 0.0^{\text {B.a }}$ & $7.8 \pm 0.1^{\text {A.a }}$ & $7.8 \pm 0.1^{\text {A.a }}$ & $7.8 \pm 0.1^{\text {A.a }}$ \\
\hline & 15 & $8.1 \pm 0.1^{\text {A.b }}$ & $8.2 \pm 0.2^{\text {A.c }}$ & $8.0 \pm 0.2^{\text {A.a }}$ & $8.1 \pm 0.0^{\text {A.ab }}$ & $8.0 \pm 0.2^{\text {A.a }}$ & $8.0 \pm 0.1^{\text {A.abc }}$ & $8.0 \pm 0.3^{\text {A.a }}$ & $7.8 \pm 0.2^{\text {A.a }}$ \\
\hline & 30 & $7.9 \pm 0.2^{\mathrm{AB} . \mathrm{ab}}$ & $7.8 \pm 0.0^{A . b}$ & $7.9 \pm 0.3^{\text {АВ.a }}$ & $8.2 \pm 0.1^{\text {B.b }}$ & $8.2 \pm 0.1^{\text {AB.a }}$ & $8.1 \pm 0.0^{A B . b c}$ & $8.1 \pm 0.2^{\text {AB.a }}$ & $8.1 \pm 0.2^{\text {AB.a }}$ \\
\hline & 45 & $7.8 \pm 0.2^{\text {A.ab }}$ & $7.8 \pm 0.0^{A . b}$ & $7.8 \pm 0.3^{\text {A.a }}$ & $7.6 \pm 0.4^{\text {A.a }}$ & $8.1 \pm 0.3^{\text {A.a }}$ & $8.2 \pm 0.2^{\text {A.c }}$ & $8.2 \pm 0.3^{\text {A.a }}$ & $8.1 \pm 0.1^{\text {A.a }}$ \\
\hline & 60 & $7.6 \pm 0.2^{\text {AB.a }}$ & $7.2 \pm 0.1^{\text {A.a }}$ & $7.6 \pm 0.5^{\text {AB.a }}$ & $7.7 \pm 0.2^{\mathrm{AB} . \mathrm{ab}}$ & $7.9 \pm 0.1^{\text {B.a }}$ & $7.8 \pm 0.1^{\mathrm{AB} . \mathrm{ab}}$ & $7.9 \pm 0.0^{\text {B.a }}$ & $8.0 \pm 0.1^{\text {B.a }}$ \\
\hline \multirow[t]{5}{*}{ Texture } & 1 & $7.9 \pm 0.1^{\text {A.b }}$ & $8.0 \pm 0.4^{A . c}$ & $7.8 \pm 0.2^{\text {A.c }}$ & $7.7 \pm 0.2^{\text {A.ab }}$ & $7.8 \pm 0.0^{\text {A.ab }}$ & $7.6 \pm 0.1^{\text {A.a }}$ & $7.5 \pm 0.3^{\text {A.a }}$ & $7.4 \pm 0.5^{\text {A.a }}$ \\
\hline & 15 & $8.2 \pm 0.1^{\mathrm{BC} . \mathrm{b}}$ & $8.0 \pm 0.2^{A B C . c}$ & $7.6 \pm 0.2^{\text {A.bc }}$ & $8.0 \pm 0.1^{\mathrm{ABC} . \mathrm{ab}}$ & $8.2 \pm 0.1^{c . b}$ & $8.0 \pm 0.0^{\mathrm{ABC} . \mathrm{a}}$ & $7 . \pm 0.4^{\mathrm{AB} . \mathrm{a}}$ & $7.8 \pm 0.2^{\text {AB.a }}$ \\
\hline & 30 & $7.1 \pm 0.2^{\text {A.a }}$ & $7.3 \pm 0.0^{\text {A.b }}$ & $7.8 \pm 0.0^{B . c}$ & $8.2 \pm 0.0^{c . b}$ & $8.3 \pm 0.1^{c . b}$ & $8.2 \pm 0.1^{\mathrm{ca}}$ & $8.2 \pm 0.1^{\text {C.a }}$ & $8.1 \pm 0.1^{\text {C.a }}$ \\
\hline & 45 & $7.1 \pm 0.0^{\text {A.a }}$ & $7.1 \pm 0.2^{A . b}$ & $7.4 \pm 0.1^{\text {AB.ab }}$ & $7.5 \pm 0.4^{\mathrm{ABC} . \mathrm{a}}$ & $7.9 \pm 0.2^{\text {BC.ab }}$ & $8.1 \pm 0.4^{\mathrm{Ca}}$ & $7.9 \pm 0.1^{\text {BC. }}$ & $7.9 \pm 0.4^{\text {BC. }}$ \\
\hline & 60 & $7.2 \pm 0.1^{\text {AB.a }}$ & $6.5 \pm 0.1^{\text {A.a }}$ & $7.2 \pm 0.1^{\text {AB.a }}$ & $7.5 \pm 0.2^{\text {AB.a }}$ & $7.5 \pm 0.5^{\text {AB.a }}$ & $7.3 \pm 1.1^{\mathrm{AB} . \mathrm{a}}$ & $7.6 \pm 0.6^{\text {AB.a }}$ & $8.0 \pm 016^{\text {B.a }}$ \\
\hline \multirow[t]{5}{*}{ Odour } & 1 & $7.2 \pm 0.0^{\mathrm{AB} . \mathrm{bc}}$ & $8.1 \pm 0.1^{\mathrm{c.d}}$ & $7.3 \pm 0.2^{\text {A.B. }}$ & $7.5 \pm 0.0^{B . c}$ & $7.5 \pm 0.1^{\mathrm{B.b}}$ & $7.1 \pm 0.1^{\text {A. } a}$ & $7.1 \pm 0.0^{\text {A. } a}$ & $7.4 \pm 0.3^{\text {AB. } a}$ \\
\hline & 15 & $7.3 \pm 0.1^{A B . c}$ & $7.9 \pm 0.1^{\text {B.cd }}$ & $7.6 \pm 0.3^{\mathrm{AB} . \mathrm{b}}$ & $7.7 \pm 0.0^{A B . d}$ & $7.5 \pm 0.0^{A B . b}$ & $7.8 \pm 0.0^{\mathrm{AB} . \mathrm{a}}$ & $7.3 \pm 0.5^{\text {AB.a }}$ & $7.6 \pm 037^{\text {A.a }}$ \\
\hline & 30 & $6.9 \pm 0.2^{\text {A.bc }}$ & $7.4 \pm 0.1^{\mathrm{B} . \mathrm{bc}}$ & $7.5 \pm 0.0^{\mathrm{B} . \mathrm{b}}$ & $7.5 \pm 0.0^{B . c}$ & $7.7 \pm 0.0^{\mathrm{B} . \mathrm{b}}$ & $7.7 \pm 0.0^{\text {B.a }}$ & $7.2 \pm 0.4^{A B . a}$ & $7.5 \pm 0.3^{\text {B.a }}$ \\
\hline & 45 & $6.8 \pm 0.4^{\mathrm{AB} . \mathrm{ab}}$ & $7.0 \pm 0.2^{\text {A.B. }}$ & $7.2 \pm 0.2^{\text {A.b. }}$ & $7.2 \pm 0.0^{\mathrm{AB} . \mathrm{b}}$ & $6.4 \pm 0.2^{\text {A.a }}$ & $7.4 \pm 0.1^{\text {B.a }}$ & $7.4 \pm 0.6^{\text {B.a }}$ & $7.2 \pm 0.2^{\text {AB.a }}$ \\
\hline & 60 & $6.4 \pm 0.0^{\text {A.a }}$ & $6.4 \pm 0.3^{\text {A.a }}$ & $6.7 \pm 0.0^{\text {A.a }}$ & $6.9 \pm 0.1^{\text {A.a }}$ & $6.0 \pm 0.5^{\text {A.a }}$ & $6.4 \pm 0.1^{\text {A.a }}$ & $6.6 \pm 0.6^{\text {A.a }}$ & $7.5 \pm 0.1^{\text {A.a }}$ \\
\hline \multirow[t]{5}{*}{ Flavour } & 1 & $7.1 \pm 0.2^{\mathrm{AB.b}}$ & $8.0 \pm 0.2^{c . b}$ & $7.2 \pm 0.2^{\mathrm{AB} . \mathrm{bc}}$ & $7.3 \pm 0.2^{\text {B.b }}$ & $7.4 \pm 0.2^{B . b}$ & $6.8 \pm 0.3^{\text {A.a }}$ & $6.8 \pm 0.2^{\text {A.ab }}$ & $7.2 \pm 0.1^{\text {AB.a }}$ \\
\hline & 15 & $6.8 \pm 0.4^{\text {A.ab }}$ & $7.4 \pm 0.0^{A B . b}$ & $7.7 \pm 0.12^{B . d}$ & $7.3 \pm 0.0^{\mathrm{AB} . \mathrm{b}}$ & $7.6 \pm 0.2^{A B \cdot b}$ & $7.7 \pm 0.6^{\text {B.a }}$ & $7.1 \pm 0.4^{\mathrm{AB} . \mathrm{B}}$ & $7.4 \pm 0.1^{\text {AB.a }}$ \\
\hline & 30 & $6.6 \pm 0.2^{\text {A.ab }}$ & $7.5 \pm 0.3^{c . b}$ & $7.2 \pm 0.1^{B C . c}$ & $6.9 \pm 0.2^{A B . a b}$ & $7.7 \pm 0.1^{c . b}$ & $7.4 \pm 0.1^{\mathrm{ca}}$ & $6.7 \pm 0.2^{\text {Aab }}$ & $7.4 \pm 0.1^{\text {ca }}$ \\
\hline & 45 & $6.6 \pm 0.0^{A B} \cdot a b$ & $6.7 \pm 0.4^{\text {AB.a }}$ & $6.8 \pm 0.2^{\text {B.ab }}$ & $6.8 \pm 0.1^{\text {Bab }}$ & $5.9 \pm 0.6^{\text {A.a }}$ & $6.5 \pm 0.7^{A B} . a$ & $7.1 \pm 0.4^{\mathrm{B} . \mathrm{b}}$ & $7.2 \pm 0.2^{\text {B.a }}$ \\
\hline & 60 & $6.4 \pm 0.0^{A B C . a}$ & $6.7 \pm 0.0^{\text {BC.a }}$ & $6.5 \pm 0.1^{\mathrm{ABC} \cdot \mathrm{a}}$ & $6.6 \pm 0.4^{\text {BCa }}$ & $5.6 \pm 0.5^{\text {Aa }}$ & $6.4 \pm 0.8^{\mathrm{ABC} \cdot \mathrm{a}}$ & $6.1 \pm 0.2^{\mathrm{AB} . \mathrm{a}}$ & $7.2 \pm 0.2^{\mathrm{Ca}}$ \\
\hline \multirow[t]{5}{*}{ Rancid taste } & 1 & $7.2 \pm 0.1^{A . c}$ & $8.3 \pm 0.0^{E . e}$ & $7.3 \pm 0.1^{\mathrm{AB} . \mathrm{b}}$ & $7.7 \pm 0.0^{C D . c}$ & $7.5 \pm 0.2^{B C D . b}$ & $7.8 \pm 0.0^{D . b}$ & $7.4 \pm 0.2^{\mathrm{ABC} . \mathrm{ab}}$ & $7.7 \pm 0.2^{\text {CD.b }}$ \\
\hline & 15 & $7.2 \pm 0.0^{\text {A.c }}$ & $8.1 \pm 0.0^{\text {B.d }}$ & $7.8 \pm 0.2^{\text {AB.b }}$ & $7.8 \pm 0.0^{A B . c}$ & $7.7 \pm 0.4^{\mathrm{B} . \mathrm{b}}$ & $7.9 \pm 0.2^{\text {B.b }}$ & $8.1 \pm 0.5^{\text {B.b }}$ & $7.9 \pm 0.1^{\mathrm{B} . b}$ \\
\hline & 30 & $7.3 \pm 0.1^{A . c}$ & $7.1 \pm 0.0^{A . C}$ & $7.2 \pm 0.4^{\text {A.b }}$ & $7.4 \pm 0.0^{\mathrm{AB} . \mathrm{b}}$ & $7.8 \pm 0.1^{B C . b}$ & $7.9 \pm 0.2^{c . b}$ & $7.7 \pm 0.1 \mathrm{~B}^{\mathrm{C}} \mathrm{ab}$ & $7.85 \pm 0.1^{B C . b}$ \\
\hline & 45 & $6.9 \pm 0.0^{\mathrm{cD} . b}$ & $6.8 \pm 0.1^{B C D . b}$ & $6.7 \pm 0.0^{\text {BC.a }}$ & $6.8 \pm 0.3^{B C D . a}$ & $5.8 \pm 0.5^{\text {A.a }}$ & $6.4 \pm 0.1^{\text {B.a }}$ & $7.0 \pm 0.1^{\mathrm{CD} . \mathrm{a}}$ & $7.2 \pm 0.1^{\text {D.a }}$ \\
\hline & 60 & $6.4 \pm 0.1^{\text {A.a }}$ & $6.3 \pm 0.0^{\text {A.a }}$ & $6.2 \pm 0.1^{\text {A.a }}$ & $6.8 \pm 0.1^{A B \cdot a}$ & $5.6 \pm 1.2^{\text {A.a }}$ & $6.3 \pm 0.1^{\text {A.a }}$ & $6.8 \pm 0.6^{A B} \cdot a$ & $7.8 \pm 0.1^{\mathrm{B} . \mathrm{b}}$ \\
\hline \multirow{5}{*}{$\begin{array}{l}\text { General } \\
\text { acceptability }\end{array}$} & 1 & $7.3 \pm 0.0^{A B C . b}$ & $8.3 \pm 0.2^{\text {D.e }}$ & $7.5 \pm 0.1^{\text {BC.b }}$ & $7.3 \pm 0.2^{\mathrm{ABC} . b c}$ & $7.6 \pm 0.0^{c . b}$ & $7.1 \pm 0.3^{\mathrm{AB} . a b}$ & $7.0 \pm 0.1^{\text {A.ab }}$ & $7.4 \pm 0.2^{\text {ABC.a }}$ \\
\hline & 15 & $7.2 \pm 0.5^{A . b}$ & $8.0 \pm 0.1^{\text {A.d }}$ & $7.9 \pm 0.2^{A . C}$ & $7.7 \pm 0.2^{A . C}$ & $7.7 \pm 0.2^{\text {A.b }}$ & $7.7 \pm 0.5^{A . b}$ & $7.5 \pm 0.4^{A . b}$ & $7.6 \pm 0.1^{\text {A.a }}$ \\
\hline & 30 & $6.7 \pm 0.1^{\text {A.ab }}$ & $7.1 \pm 0.1^{B . c}$ & $7.3 \pm 0.1^{\mathrm{BC} . \mathrm{b}}$ & $7.4 \pm 0.0^{c . b c}$ & $7.8 \pm 0.1^{\mathrm{D} . b}$ & $7.7 \pm 0.1^{\mathrm{D} . b}$ & $7.2 \pm 0.1^{\text {B.ab }}$ & $7.8 \pm 0.1^{\text {D.a }}$ \\
\hline & 45 & $6.7 \pm 0.1^{\text {AB.ab }}$ & $6.7 \pm 0.1^{\mathrm{AB} . \mathrm{b}}$ & $6.6 \pm 0.2^{\mathrm{AB} . \mathrm{a}}$ & $7.1 \pm 0.1^{\text {B.ab }}$ & $6.2 \pm 0.8^{\text {A.a }}$ & $6.9 \pm 0.5^{\mathrm{AB} . \mathrm{ab}}$ & $7.3 \pm 0.1^{\text {B.b }}$ & $7.5 \pm 0.0^{\text {B.a }}$ \\
\hline & 60 & $6.2 \pm 0.4^{\mathrm{AB} . \mathrm{a}}$ & $6.2 \pm 0.0^{\mathrm{AB} . a}$ & $6.3 \pm 0.0^{A B} \cdot a$ & $6.8 \pm 0.0^{B C . a}$ & $5.9 \pm 0.5^{\text {A.a }}$ & $6.6 \pm 0.4^{A B C . a}$ & $6.5 \pm 0.4^{\mathrm{ABC} . \mathrm{a}}$ & $7.3 \pm 0.4^{c . a}$ \\
\hline
\end{tabular}

YA: yogurt butter control; YB: yogurt butter produced with L. lactis subsp. lactis biovar. diacetylactis (2 \%); YC: yogurt butter produced with Leu. mesenteroides subsp. cremoris (2\%); YD: yogurt butter produced with Leu. mesenteroides subsp. cremoris (1 \%) + L. lactis subsp. lactis biovar. diacetylactis (1 \%); CA: cream butter control; CB: cream butter produced with L. lactis subsp. lactis biovar. diacetylactis (2 \%); CC: cream butter produced with Leu. mesenteroides subsp. cremoris (2\%); CD: cream butter produced with Leu. mesenteroides subsp. cremoris (1\%) + L. lactis subsp. lactis biovar. diacetylactis (1\%), lower letters in a row (same samples in different storage days) and capital letters (different samples in same storage days) with no common superscript differ $(P<0.05)$.

control butter. During sensory evaluation, sample D was selected as the most popular butter by the assessors in terms of the general acceptability. It was showed that the use of starters or storage time significantly influenced the SPME/GC-MS volatile fraction and sensory characteristics of the samples. Bacteria of the genus Leuconostoc are used in dairy industry as they produce carbon dioxide and flavour compounds by the heterofermentative utilization of lactose and citrate. The production of volatile compounds in butter such as diacetyl or acetoin, which are produced by citrate and sugar co-metabolism (Schmitt et al., 1997). In this context, use of starters in production and storage of the samples caused significant effects on the formation of diacetyl, butyric and hexanoic acids.

Morgan et al. (1966) noted that malty compounds are metabolites of specific amino acids, which are deaminated and decarboxylated. For example, leucine is metabolised to 3-methy-1-butanal 
and the corresponding alcohol; isoleucine is metabolised to 2-methyl-1-butanal/butanol; and finally valine is metabolised to 2-methyl-1-propanal/propanol (Narvhus et al., 1998). In this study, these compounds were found in high concentrations in cream butters because the levels of fat-free substance (Table 1) were higher in cream butters than yogurt butters.

\section{Conclusion}

In this study, the use of starter culture in the manufacture of the two types of butter (yogurt or cream butter types) was discussed during storage. The numbers of volatile compounds of cream butter were higher than those of yogurt butter. The principal component analysis of data showed that the samples were primarily grouped based on raw material (cream or yogurt) and then according to storage time. After sensory evaluation of samples, butter samples produced with starter cultures had higher scores than the non-starter containing control sample. The highest general acceptability scores were given for the butter samples containing mixed cultures at the end of storage. The results showed that the use of starter culture in the manufacture of yogurt butter, which is traditionally produced without starters, significantly affected the volatile composition and sensory properties. In conclusion, it is suggested that starter D containing Leu. mesenteroides subsp. cremoris + L. lactis subsp. lactis biovar. diacetylactis was suitable for producing yogurt and cream butter with the best volatile characteristics. However, further studies need to be conducted to identify the best culture and conditions for yogurt butter.

\section{Acknowledgements}

The authors thank the Atatürk University Research Fund for supporting this study (BAP Project No: 2009/201).

\section{Utjecaj starter kulture i skladištenja na hlapive sastojke i senzorska svojstva maslaca od vrhnja i maslaca od jogurta}

\section{Sažetak}

U ovom istraživanju ispitivan je utjecaj starter kulture na hlapive sastojke i senzorska svojstva maslaca proizvedenog od vrhnja, odnosno od jogurta tijekom 60 dana hladnog skladištenja. Kao starter kulture korišteni su sojevi Lactococcus lactis subsp. lactis biovar. diacetylactis, Leuconostoc mesenteroides subsp. cremoris te njihova kombinacija. Utvrđeno je formiranje ukupno 10 estera, 6 aldehida, 11 ketona, 16 alkohola, 6 kiselina, 2 sulfida, 3 terpena i 3 atipična sastojka. U pravilu je maslac od vrhnja sadržavao više hlapivih sastojaka nego maslac od jogurta. Prisutnost S-metil tioacetata utvrđena je samo u maslacu od jogurta, dok su u maslacu od vrhnja bili prirodno prisutni heksanal, etanol, 2-nonanon, 2-pentanon, 2-heptanon, acetat, maslačna i kapronska kiselina. lako se maslac od jogurta inače proizvodi bez startera, dobiveni rezultati su pokazali kako bi korištenje starter kultura u proizvodnji ovog tipa maslaca pozitivno utjecalo na stvaranje hlapivih sastojaka te na njegova senzorska svojstva. Maslac proizveden korištenjem kombinirane starter kulture dobio je najviše ocjene za prihvatljivost.

\section{Ključne riječi: maslac od jogurta, maslac od vrhnja, starter kultura, hlapivi sastojci, senzorska svojstva, skladištenje}


1. Altuntas, S., Korukluoglu, M. (2019): The impact of different commercial probiotic cultures with starters on technological, physicochemical and sensorial properties of a traditional yogurt-based appetizer "Cacık". Mljekarstvo 69 (3), 193-205. https://doi: 10.15567/mljekarstvo.2019.0305

2. Ayad, E.H., Verheul, A., Engels, W.J., Wouters, J., Smit, G. (2001): Enhanced flavour formation by combination of selected lactococci from industrial and artisanal origin with focus on completion of a metabolic pathway. Journal of Applied Microbiology 90, 59-67. https://doi.org/10.1046/j.1365-2672.2001.01219.x

3. Ayar, A., Sert, D., Arslan, D., Özcan, M.M. (2010): The effect of some spice extracts on storage stability of "Yayik butter". World Applied Sciences Journal, 11, 1114-1123. https://doi.org/10.2376/0003-925X-66-10

4. Beshkova, D., Simova, E., Frengova, G., Simov, Z. (1998): Production of flavour compounds by yogurt starter cultures. Journal of Industrial Microbiology and Biotechnology 20, 180-186. https://doi.org/10.1038/sj.jim.2900504

5. Ciganek, M., Neca, J. (2008): Chemical characterization of volatile organic compounds on animal farms. Veterinarni Medicina 53, 641-651.

6. Clark, S., Winter, C.K. (2015): Diacetyl in foods: A review of safety and sensory characteristics. Comprehensive Reviews in Food Science and Food Safety, 14, 634-643. https://doi: 10.1111/1541-4337.12150

7. Couvreur, S., Hurtaud, C., Lopez, C., Delaby, L., Peyraud, J.L. (2006): The linear relationship between the proportion of fresh grass in the cow diet, milk fatty acid composition, and butter properties. Journal of Dairy Science 89, 19561969. https://doi.org/10.3168/jds.S0022-0302(06)72263-9

8. Çakir, Y., Cakmakçi, S., Hayaloglu, A.A. (2016): The effect of addition of black cumin (Nigella sativa L.) and ripening period on proteolysis, sensory properties and volatile profiles of Erzincan Tulum (Savak) cheese made from raw Akkaraman sheep's milk. Small Ruminant Research 137,63-74.

https://doi.org/10.1016/j.smallrumres.2015.12.004

9. Çakmakçı, S., Gündoğdu, E., Dağdemir, E., Erdoğan, Ü. (2014): Investigation of the possible use of black cumin (Nigella sativa L.) essential oil on butter stability. Kafkas Universitesi Veteriner Fakultesi Dergisi 20, 533-539. https://doi.org/10.9775/kvfd.2018.21598

10. Demirkol, A., Güneşer, O., Karagül Yüceer, Y. (2016): Volatile compounds, chemical and sensory properties of butters sold in Çanakkale. Tarım Bilimleri Dergisi - Journal of Agricultural Sciences 22, 99-108.

11. Edris, A., Schirle-Keller, J. (2010): Composition and aroma impact description of volatile components of farmer's sour cream butter using GC-MS-olfactometry. Arab Universities Journal of Agricultural Sciences 18,165-170.

12. Engels, W.J.M.Dekker, R., De Jong, C., Neeter, R., Visser, S. (1997). A comparative study of volatile compounds in the water-soluble fraction of various types of ripened cheese. International Dairy Journal, 7, 255-263. https://doi.org/10.1016/S0958-6946(97)00003-4
13. Fox, P.F., Wallace, J.M. (1997): Formation of flavour compounds. Advances in Applied Microbiology 45, 17-85.

14. Gürkan, H., Hayaloglu, A.A. (2017): Volatiles and sensory characteristics of yogurt manufactured by incorporating basil (Ocimum basilicum L.). International Journal of Food Properties 20, 779-789. https://doi.org/10.1080/10942912.2017.1311344

15. Hayaloğlu, A.A., Konar, A. (2001): Comparative study on physicochemical and sensorial properties of butter made from yoghurt and cream. Milchwissenschaft 56, 675-677.

16. Kaminarides, S., Stamou, P., Massouras, T. (2007): Comparison of the characteristics of set type yoghurt made from ovine milk of different fat content. International Journal of Food Science and Technology 42, 1019-1028. https://doi.org/10.1111/j.1365-2621.2006.01320.x

17. Kaminarides, S., Nestoratos, K., Massouras, T. 2013 : 'Effect of added milk and cream on the physicochemical, rheological and volatile compounds of Greek whey cheeses. Small Ruminant Research 113 (2-3), 446-453. https://doi.org/10.1016/j.smallrumres.2013.04.009

18. Karaca, Y., Gün, İ., Seydim A.C., Guzel-Seydim, Z.B. (2018): Production and quality of kefir cultured butter. Mljekarstvo 68 (1), 64-72.

19. Karatapanis, A.E., Badeka, A.V., Riganakos, K.A., Savvaidis, I.N., Kontominas, M.G. (2006): Changes in flavour volatiles of whole pasteurized milk as affected by packaging material and storage time. International Dairy Journal 16, 750-761. https://doi.org/10.1016/j.idairyj.2005.06.008

20. Liu, S.Q., Asmundson, R.V., Holland, R., Crow, V.L. (1997): Acetaldehyde metabolism by Leuconostoc mesenteroides subsp. cremoris under stress conditions. International Dairy Journal 7, 175-183.

https://doi.org/10.1016/S0958-6946(96)00055-6

21. López-González, M.J., Escobedo, S., Rodríguez, A., Neves, R.T., Janzen, T., Martínez, B. (2018): Adaptive evolution of industrial Lactococcus lactis under cell envelope stress provides phenotypic diversity. Frontiers in Microbiology 9, 1-17. https://doi.org/10.3389/fmicb.2018.02654

22. Lozano, P.R., Miracle, E.R., Krause, A.J., Drake, M., Cadwallader, K.R. (2007): Effect of cold storage and packaging material on the major aroma components of sweet cream butter. Journal of Agricultural and Food Chemistry 55, 7840-7846. https://doi.org/10.1021/jf071075q

23. Mallia, S., Fernández-García, E., Bosset, J.O. (2005): Comparison of purge and trap and solid phase microextraction techniques for studying the volatile aroma compounds of three European PDO hard cheeses. International Dairy Journal 15, 741-758. https://doi.org/10.1016/j.idairyj.2004.11.007

24. Marsili, R., Miller, N. (1998): Determination of the cause of off-flavors in milk by dynamic headspace GC/MS and multivariate data analysis. Developments in Food Science 40, 159-171. https://doi.org/10.1016/S0167-4501(98)80042-3 
25. McSweeney, P.L.H, Sousa, M.J. (2000). Biochemical pathways for the production of flavour compounds in cheese during ripening: A review. Lait 293-324. https://doi.org/10.1051/lait:2000127

26. Morgan, M.E., Lindsay, R.C., Libbey, L.M. (1966): Identity of additional aroma constittuents in milk cultures of Streptococcus lactis var. maligenes. Journal of Dairy Science 49, 5-18. https://doi.org/10.3168/jds.S0022-0302(66)87777-9

27. Mutukumira, A.N. (1996): Investigation of some prospects for the development of starter cultures for industrial production of traditional fermented milk in Zimbabwe. Norges Landbrukshoegskole, Aas (Norway).

28. Nájera-Domínguez, C., Gutiérrez-Méndez, N., NevárezMoorillon, G., Caro-Canales, I. (2014): Comparison of volatile compounds produced by wild Lactococcus lactis in miniature Chihuahua-type cheeses. Dairy Science and Technology 94, 499-516. https://doi.org/10.1007/s13594-014-0175-4

29. Narvhus, J., Østeraas, K., Mutukumira, T., Abrahamsen, R. (1998): Production of fermented milk using a malty compound-producing strain of Lactococcus lactis subsp. lactis biovar. diacetylactis, isolated from Zimbabwean naturally fermented milk. International Journal of Food Microbiology 41, 73-80. https://doi.org/10.1016/S0168-1605(98)00036-1

30. Ozcan, T., Akpinar-Bayizit, A, Yilmaz-Ersan, L., Cetin K., Delikanli, B. (2016): Evaluation of fatty acid profile of Trabzon butter. International Journal of Chemical Engineering and Applications 7 (3), 190-194. https://doi.org/10.7763/IJCEA.2016.V7.570

31. Pawliszyn, J., Pawliszyn, B., Pawliszyn, M. (1997): Solid phase microextraction (SPME). The Chemical Educator 2, 1-7. https://doi.org/10.1007/s00897970137a

32. Peterson, D, Reineccius, G. (2003): Determination of the aroma impact compounds in heated sweet cream butter. Flavour and Fragrance Journal 18, 320-324. https://doi.org/10.1002/ff.1228

33. Puniya, A.K. (2016): Fermented Milk and Dairy Products. CRC Press, Taylor \& Francis Group, Boca Raton.

34. Reineccius, G. (2006): Flavor Chemistry and Technology. In Book Flavor Chemistry and Technology. Series Flavor Chemistry and Technology. Taylor \& Francis Group, New York.
35. Ruiz Rodríguez, L.G., Mohamed, F., Bleckwedel, J., Medina, R. (2019): Diversity and functional properties of lactic acid bacteria isolated from wild fruits and flowers present in Northern Argentina. Frontiers in Microbiology 10, 1091. https://doi.org/10.3389/fmicb.2019.01091

36. Sağdiç, O., Arici, M., Simşek, O. (2002): Selection of starters for a traditional Turkish yayik butter made from yoghurt. Food Microbiology 19, 303-312. https://doi.org/10.1006/fmic.2002.0489

37. Sağdıç, O., Dönmez, M., Demirci, M. (2004): Comparison of characteristics and fatty acid profiles of traditional Turkish yayik butters produced from goats', ewes' or cows' milk. Food Control 15, 485-490. https://doi.org/10.1016/j.foodcont.2003.07.003

38. Schmitt, P., Vasseur, C., Phalip, V., Huang, D., Divies, C., Prevost, H. (1997): Diacetyl and acetoin production from the co-metabolism of citrate and xylose by Leuconostoc mesenteroides subsp. mesenteroides. Applied Microbiology and Biotechnology 47, 715-718. https://doi.org/10.1007/s002530051000

39. Şenel, E, Atamer, M., Öztekin, F.Ş. (2011): The oxidative and lipolytic stability of Yayık butter produced from different species of mammals milk (cow, sheep, goat) yoghurt. Food Chemistry 127, 333-339. https://doi.org/10.1016/j.foodchem.2011.01.012

40. Tamime, A.Y., Robinson, R.K. (2007): Tamime and Robinson's Yoghurt: Science and Technology, 3rd edition. Woodhead Publishing, ISBN: 978-1-84569-213-1.

41. Valdivielso, I., de Renobales, M., Aldai, N., Baron, L.J.R. (2017): Changes in terpenoid composition of milk and cheese from commercial sheep flocks associated with seasonal feeding regimens throughout lactation. Journal of Dairy Science 100, (1), 96-105. https://doi.org/10.3168/jds.2016-11761

42. Yahia, E.M. (2017): Fruit and Vegetable Phytochemicals: Chemistry and Human Health, 2 Vol. John Wiley \& Sons.

43. Yu,T.-H., Ho., C.-T. (1995). Volatile compounds generated from thermal reaction of methionine and methionine sulfoxide with or without glucose. Journal of Agricultural and Food Chemistry. 43, 1641-1646. 